\title{
Structure Functions in Deep Inelastic Lepton-Nucleon Scattering
}

\author{
Max Klein \\ DESY/Zeuthen, Platanenallee 6, D-15738 Zeuthen_klein@ifh.de
}

\begin{abstract}
Latest results on structure functions, as available at the Lepton-Photon Symposium 1999, are presented. This report focusses on three experimental areas: new structure function measurements, in particular from HERA at low $x$ and high $Q^{2}$; results on light and heavy flavour densities; determinations of the gluon distribution and of $\alpha_{s}$. As the talk was delivered at a historic moment and place, a few remarks were added recalling the exciting past and looking into the promising future of deep inelastic scattering (DIS).
\end{abstract}

\section{Introduction}

About three decades ago, highly inelastic electron-proton scattering was observed by a SLAC-MIT Collaboration [1] which measured the proton structure function $\nu W_{2}\left(Q^{2}, \nu\right)$ to be independent of the four-momentum transfer squared $Q^{2}$ at fixed Bjorken $x=Q^{2} / 2 M_{p} \nu$. Here $\nu=E-E^{\prime}$ is the energy transferred by the virtual photon. It is related to the inelasticity $y$ through $\nu=s y / 2 M_{p}$, with proton mass $M_{p}$ and the energy squared in the centre of mass system $s=2 M_{p} E$. With the SLAC linear accelerator the incoming electron energy $E$ had been successfully increased by a factor of twenty as compared to previous form factor experiments [2]. Thus $Q^{2}=4 E E^{\prime} \sin ^{2}(\theta / 2)$ could be enlarged and measured using the scattered electron energy $E^{\prime}$ and its polar angle $\theta$. Partonic proton substructure [3] was established at $1 / \sqrt{Q^{2}} \simeq 10^{-16} \mathrm{~m}$ which allowed the scaling behaviour [4] of $\nu W_{2}\left(Q^{2}, \nu\right) \rightarrow F_{2}(x)$ to be interpreted. In the quark-parton model (QPM) [5] the structure function $F_{2}$ is given by the momentum distributions of valence and sea quarks, $q=q_{v}+q_{s}$, and of antiquarks $\bar{q}$ weighted by the square $Q_{q}^{2}$ of the electric charge, $F_{2}\left(x, Q^{2}\right)=x \sum_{q} Q_{q}^{2}(q+$ $\bar{q})$. Neutrino experiments found $\sigma_{\nu} \simeq 3 \sigma_{\bar{\nu}}$ demonstrating that partons could be identified with quarks having gauge couplings like leptons and that at large $x$ the sea is small. Scaling violations were hidden in the first DIS data taken at $x \simeq 0.2$, as if we needed help to understand the basics of inelastic scattering. They were found in $\mu N$ scattering [6] in an extended $x, Q^{2}$ range. The logarithmic $Q^{2}$ dependence of $F_{2}\left(x, Q^{2}\right)$, established in subsequent neutrino and muon-nucleon scattering experiments, was attributed to quark-gluon interactions in Quantum Chromodynamics [7]. 


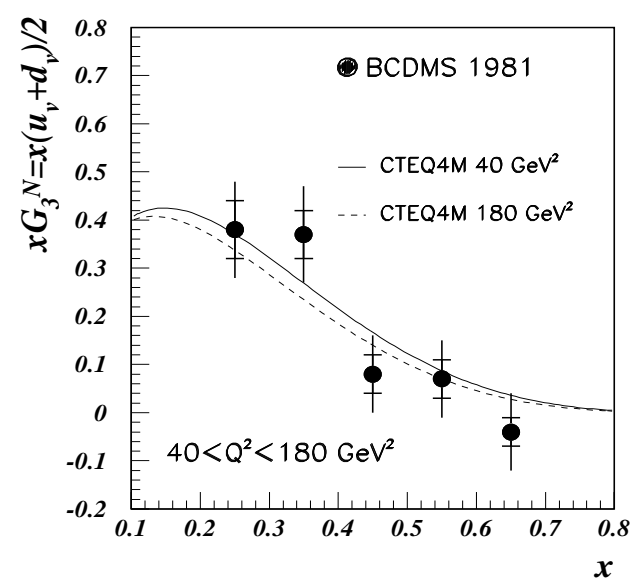

Figure 1: Measurement of the $\gamma Z$ interference structure function $x G_{3}$ in $\mu^{ \pm}$Carbon scattering by the BCDMS Collaboration compared with a recent parametrization of the valence quark distributions by the CTEQ group.

With the discovery of neutral currents [8] DIS neutrino experiments made a major contribution to the theory of weak interactions. In 1979 another ep scattering experiment was performed at SLAC [9] which determined in a highly sensitive polarization asymmetry measurement at $Q^{2} \simeq 1.5 \mathrm{GeV}^{2}$ the right-handed weak isospin charge of the electron to be zero. This experiment selected thus the Glashow Weinberg Salam model as the standard electroweak theory and opened the possibility to investigate proton structure at high $Q^{2}$ via $Z$ boson exchange. The nucleon structure function $F_{2}$ was generalized, still in a $V-A$ theory [10], to three functions

$$
\left(F_{2}, G_{2}, H_{2}\right)=x \sum_{q}\left(Q_{q}^{2}, 2 Q_{q} v_{q}, v_{q}^{2}+a_{q}^{2}\right)(q+\bar{q})
$$

arising from photon exchange $\left(F_{2}\right), \gamma Z$ interference $\left(G_{2}\right)$ and $Z$ exchange $\left(H_{2}\right)$, where $v_{q}\left(a_{q}\right)$ are the vector (axial vector) quark couplings [11]. In charged lepton-nucleon neutral current $(\mathrm{NC})$ scattering two further structure functions appear which are analogous to $x F_{3}$ in neutrino scattering

$$
\left(x G_{3}, x H_{3}\right)=2 x \sum_{q}\left(Q_{q} a_{q}, v_{q} a_{q}\right)(q-\bar{q}) .
$$

A DIS muon experiment with simultaneous beam charge and polarity reversal resulted in the first determination of the $\gamma Z$ interference structure function $x G_{3}$ at $Q^{2} \simeq$ $60 \mathrm{GeV}^{2}$ by the BCDMS Collaboration at CERN, Fig. 目. Electroweak interference occurs at the level of $\kappa \simeq 10^{-4} Q^{2} / \mathrm{GeV}^{2}$ as defined by the ratio of the weak and the electromagnetic coupling constants. Since the axial vector couplings could be considered to be known this was an interesting measurement of the valence quark 
distribution sum $u_{v}+d_{v}$ which confirmed the sign of the quark charge combination $Q_{u}-Q_{d}$ to be positive.

With the HERA energy of $s=4 E_{e} E_{p} \simeq 10^{5} \mathrm{GeV}^{2}$ the kinematic range of DIS experiments could be greatly extended towards high $Q^{2}$ since $s$ was enlarged by a factor of about $2 E_{p} / \mathrm{GeV}$ compared to fixed target scattering. The first measurements of $F_{2}$ by the H1 [13] and the ZEUS [14] Collaborations, using data taken in 1992, reached $x \simeq 0.0005$ at $Q^{2} \simeq 20 \mathrm{GeV}^{2}$. They discovered a steep rise of $F_{2}\left(x, Q^{2}\right)$ towards low $x$ at fixed $Q^{2}$ : below $x \simeq 0.01$ a decrease by one order of magnitude translates into an increase of $F_{2}$ by about a factor of two, Fig. 2. Although a "Possible
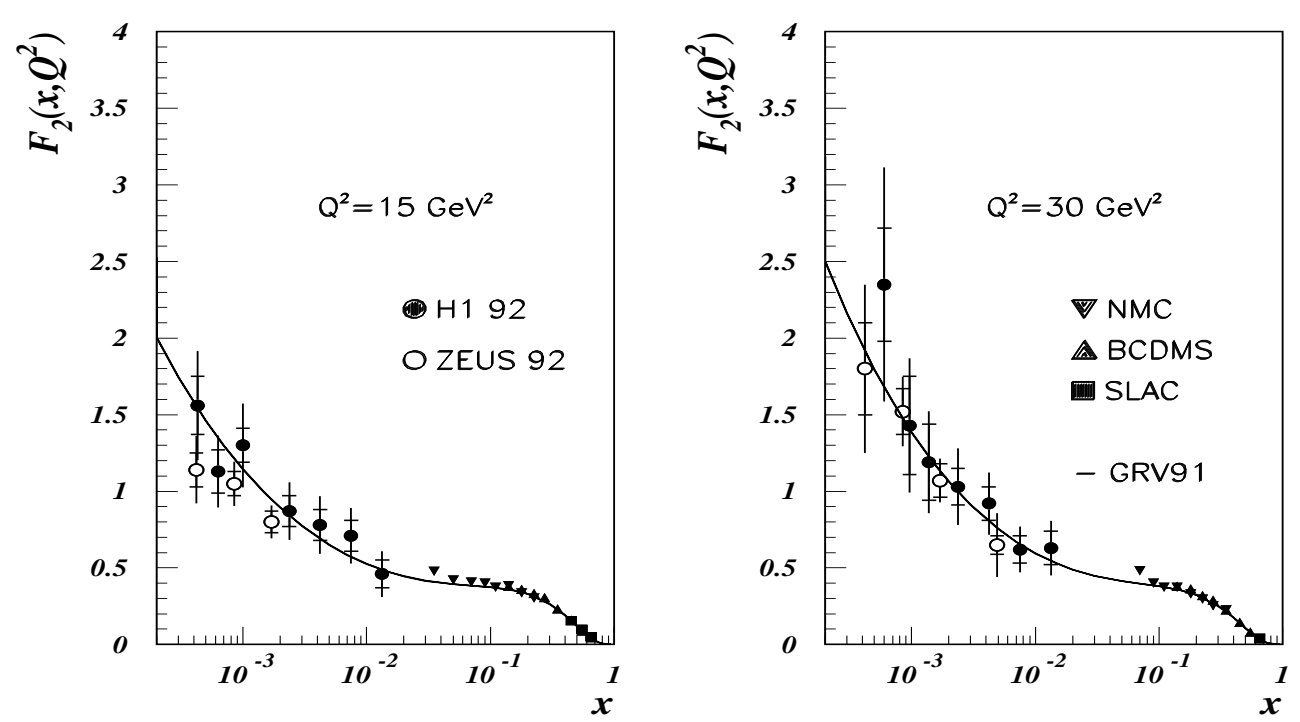

Figure 2: Measurements of the proton structure function $F_{2}\left(x, Q^{2}\right)$ by the muonproton scattering experiments BCDMS and NMC and their extension towards low $x$ by the first available HERA data on $F_{2}$. The curve represents the anticipation of this rising behaviour by Glück, Reya and Vogt [17.

Non-Regge Behaviour of Electroproduction Structure Functions" [15 at low $x$ had been considered and the concept [16 and modified phenomenology [17 of 'dynamical partons' had been worked out, this rise came as some surprise since the DGLAP evolution equations do not a priori fix the $x$ behaviour. This rise is now basically understood as being due to the dominance of gluons which leads to the description of the scaling violations as $\partial F_{2} / \partial \ln Q^{2} \propto \alpha_{s} \cdot x g$ for $Q^{2} \gg M_{p}^{2}$ and low $x$. Its quantitative description in NLO QCD and the search for new dynamics 18 connected with large logarithms of $1 / x$ requires highest possible precision, i.e. improved instrumentation and higher luminosity than was available when the first observation was made.

While much attention has been paid to the inclusive and charm structure function measurements at HERA, remarkable progress was also achieved in the investigation 
of up, down, strange and charm quark distributions with neutrino and Drell-Yan experiments at the Tevatron.

This paper describes a talk on structure functions in deep-inelastic scattering delivered in 1999. Such a report is to some extent personal and cannot possibly cover this expanding field of particle physics in any exhaustive fashion. It thus may be seen together with further articles, e.g. [19, 20], and with the conference on deep inelastic scattering and QCD held at Zeuthen in April 1999 [21]. It demonstrates remarkable progress in DIS since the previous Symposium on Lepton-Photon Interactions [22]. This talk focussed on recent measurements of structure functions (Section 2), of quark distributions including charm (Section 3) and determinations of the gluon distribution and of $\alpha_{s}$ (Section 4). The field of deep inelastic lepton-nucleon scattering has an exciting future as will be described briefly in Section 5 .

\section{Recent Measurements of Structure Functions}

Since the first SLAC experiment, fixed target muon and neutrino-nucleon scattering experiments and subsequently the HERA collider experiments H1 and ZEUS extended the explored kinematic region of DIS by several orders of magnitude, Fig. 3 . At smallest $x$ partons carry only a vanishing fraction of the proton momentum. Hence the kinematics resembles the fixed target experiments where both the electron and hadrons are scattered into the lepton beam direction (unfortunately termed 'backward' at HERA). For high $Q^{2}>s x E_{e} /\left(E_{e}+x E_{p}\right)$, i.e. $Q^{2}>2,800 \mathrm{GeV}^{2}$ for $x>0.5$, the electron is scattered through angles $\theta>90^{\circ}$ with respect to the electron beam direction, similar to Rutherford backscattering. The kinematic range of the HERA collider experiments is confined to about $y \geq 0.001$. For lower $y$ hadrons escape in the forward (proton beam) direction. At very small $y$ the inclusive kinematics cannot be reliably reconstructed using the scattered electron variables alone since the $x$ resolution varies like $1 / y$.

Until 1997 HERA ran with positrons scattered off protons of $820 \mathrm{GeV}$ energy and about $40 \mathrm{pb}^{-1}$ of luminosity became available for each collider experiment. From 1998 till May 1999 data samples of about $15 \mathrm{pb}^{-1}$ were collected in collisions of electrons with $920 \mathrm{GeV}$ protons. The $e^{ \pm}$energy is tuned to about $27.5 \mathrm{GeV}$ to optimize the polarization for the fixed target experiment HERMES. Longitudinal lepton beam polarization is foreseen to be used in colliding beam mode from 2001 onwards.

\subsection{Transition to Photoproduction and Low $Q^{2} \leq M_{p}^{2}$}

The structure function $F_{2}$ which dominates the DIS cross section behaves like $x^{-\lambda\left(x, Q^{2}\right)}$ and vanishes due to gauge invariance with $Q^{2} \rightarrow 0$ like $\mathrm{O}\left(Q^{2}\right)$. The total virtual photon-proton scattering cross section is related to $F_{2}$ as $\sigma_{\text {tot }}^{\gamma^{*} p} \simeq 4 \pi^{2} \alpha \cdot F_{2} / Q^{2}$. Mea- 


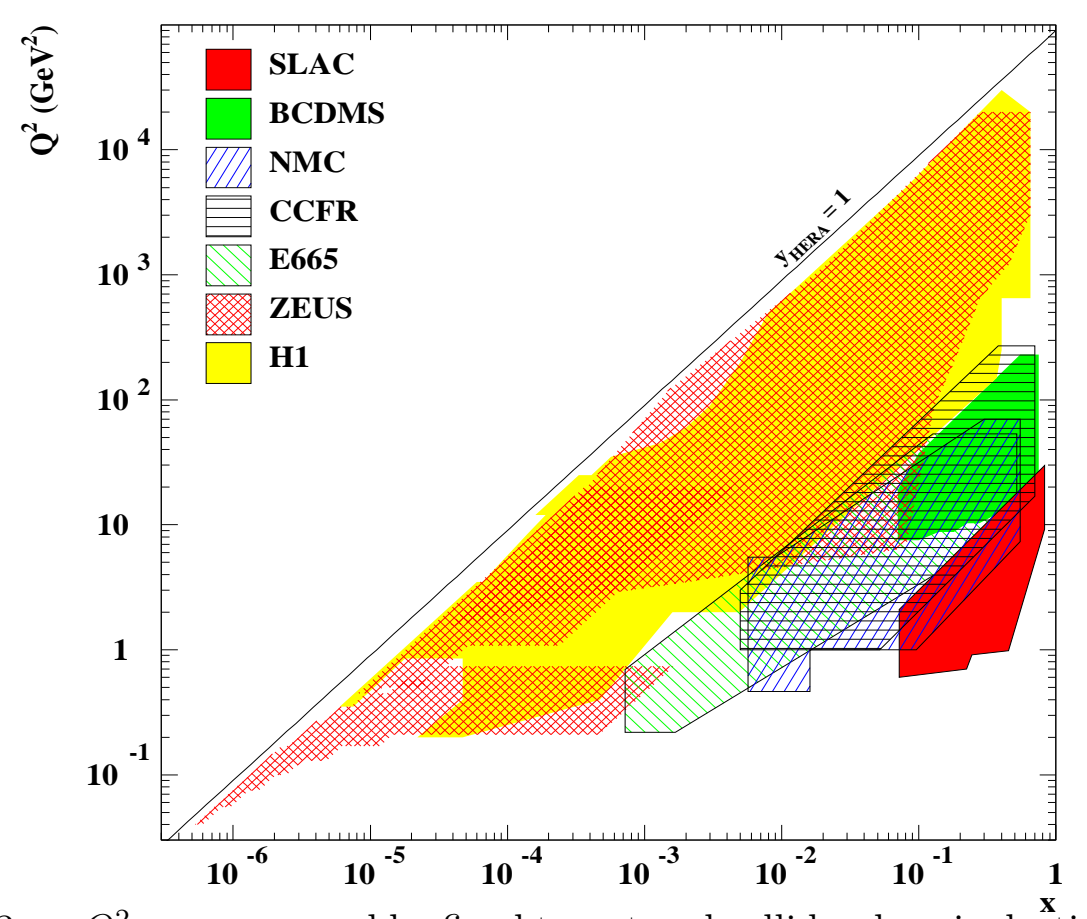

Figure 3: $x, Q^{2}$ range covered by fixed target and collider deep inelastic neutral current scattering experiments carried out until 1997.

surements of $F_{2}$ at low $Q^{2}$ investigate the dynamics of the transition from the deep inelastic to the photoproduction regime [23]. In Regge theory the structure function $F_{2}$ results from a superposition of exchanged Regge poles with intercepts $\alpha_{i}$, $F_{2}=\sum \beta_{i}\left(Q^{2}\right) W^{2 \alpha_{i}-2}$, where $W^{2} \simeq Q^{2} / x \gg Q^{2}$ for low $x, W$ being the invariant mass of the $\gamma^{*} p$ system. A recent fit to $F_{2}$ data (DL98) is rather successful using three trajectories, i.e. $\alpha_{1}=1.08$ for the soft pomeron, $\alpha_{2}=0.55$ for $a$ and $f$ exchange and $\alpha_{3}=1.4$ for the so-called hard pomeron [24]. For $Q^{2} \rightarrow 0$ the exponent $\lambda$ is approximately given by the dominant pomeron Regge trajectory, i.e. $\lambda \simeq \alpha_{1}-1 \simeq 0.1$. The recent ZEUS data [25], obtained with a backward calorimeter and tracker positioned close to the beam pipe, are rather well described by this model, Fig. *. Phenomenological models using a combination of Generalized Vector Meson Dominance [26] and perturbative QCD [27] describe this transition also well. Extrapolations of $F_{2}\left(x, W^{2}\right)$ to $Q^{2} \simeq 0$ come out to be somewhat higher than the direct measurements of $\sigma_{\text {tot }}^{\gamma^{*} p}$ 25] with tagged electrons. The $F_{2}$ based $\sigma_{\text {tot }}^{\gamma^{*} p}$ data are still at some $Q^{2}$ distance from the real photoproduction measurements which have uncertainties of about $10 \%$ due to beam optics and the imperfect simulation of the complete final state. Further extension of the range of the inclusive $F_{2}$ measurements at HERA towards lowest $Q^{2}$ values is thus desirable. This could be achieved in a rather short run of HERA at minimum possible electron beam energy since $Q^{2}$ is proportional to $E^{2}$ for all except the high $y$ values. 


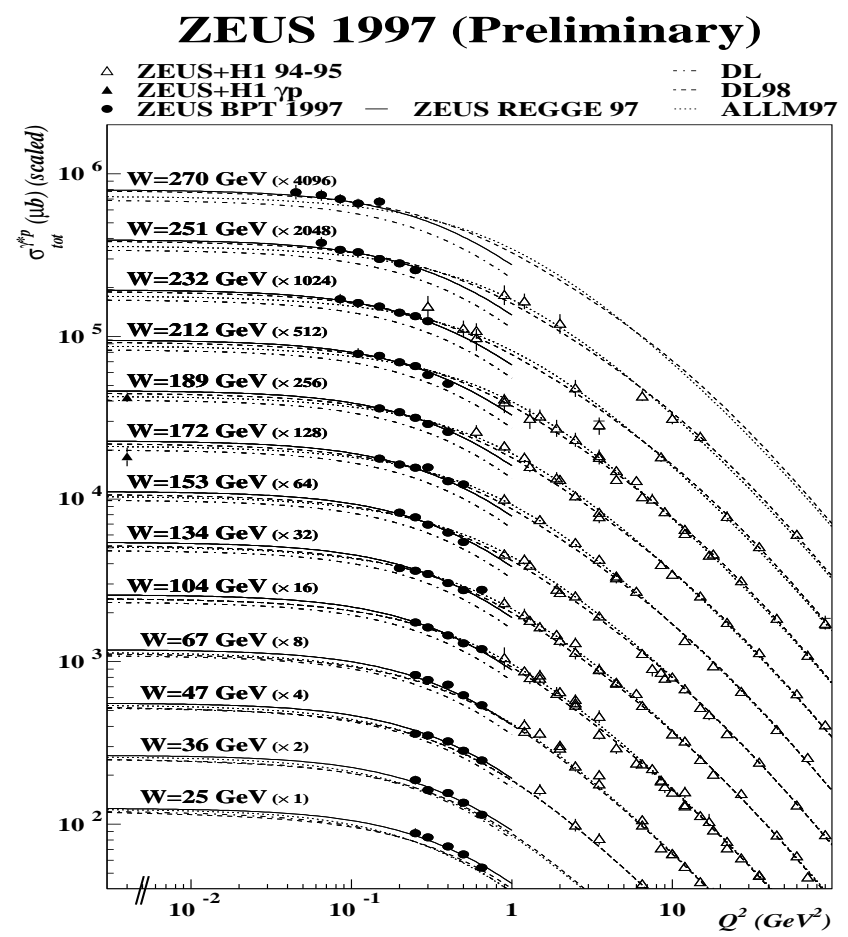

Figure 4: Measurements of the proton structure function $F_{2}\left(x, Q^{2}\right)$ expressed as $\sigma_{\text {tot }}^{\gamma^{*} p}\left(Q^{2}, W^{2}\right)$ from recent low $Q^{2}$ data by ZEUS (closed points) and rebinned $F_{2}$ data at larger $Q^{2}$ by $\mathrm{H} 1$ and ZEUS (open squares). The two triangles at $W=172 \mathrm{GeV}$ and $W=189 \mathrm{GeV}$ near $Q^{2} \approx 0$ denote the direct photoproduction cross section measurements of $\mathrm{H} 1$ and ZEUS.

New data on parton-hadron duality [28] became available this year from an experiment at Jefferson Laboratory [29] measuring electron-proton and deuteron scattering in the resonance region $W \simeq 1 \mathrm{GeV}$. The superposition of cross sections, determined at different $Q^{2}$ between 0.2 and $3.3 \mathrm{GeV}^{2}$, leads to an averaged behaviour of $F_{2}$ which is valence like even at low $x$, or mass corrected $\xi$ [30], which supports the assumption made in the GRV analysis [17] for the initial $x$ distributions at very small $Q^{2}$. In this experiment, which in the future will measure the ratio $R=\sigma_{L} / \sigma_{T}$, one estimates power corrections ('higher twists') to be small and derives the magnetic elastic proton form factor $G_{M}^{p}$ from inelastic data.

\subsection{Neutrino Experiments}

The final measurement of $\nu F e$ and $\bar{\nu} F e$ scattering cross sections by the CCFR Collaboration [31] is in good agreement with previous data obtained by the CDHSW Collaboration and more accurate. The high statistics CCFR data has been used for a number of investigations regarding all structure functions involved (Sections 2.3, 2.4) 
and also for tests of QCD (Section 4.3). Recently data were released for extremely large $x>0.75$ pointing to cumulative effects beyond Fermi motion in the nucleus [32] which were studied previously by the BCDMS Collaboration [33.

Data were obtained by the IHEP-JINR neutrino experiment in the wide band neutrino beam at the Serpukhov U70 accelerator [34]. Based on about $750 \nu$ and $6000 \bar{\nu}$ events for $W^{2}>1.7 \mathrm{GeV}^{2}$ and $Q^{2} \simeq 2 \mathrm{GeV}^{2}$, the structure functions $F_{2}$ and $x F_{3}$ were disentangled and $\alpha_{s}\left(M_{Z}^{2}\right)=0.123_{-0.013}^{+0.010}$ was determined in NLO QCD.

\subsection{Precision Measurement at Low $x$ and Medium $Q^{2}$}

The H1 Collaboration released for this conference the so far most precise measurement of the DIS cross section at HERA. In its reduced form it can be written as

$$
\frac{Q^{4} x}{2 \pi \alpha^{2} Y_{+}} \cdot \frac{d^{2} \sigma}{d Q^{2} d x}=\sigma_{r}=F_{2}-\frac{y^{2}}{Y_{+}} \cdot F_{L},
$$

i.e. $\sigma_{r} \simeq F_{2}$ apart from high $y$ where $\sigma_{r} \rightarrow F_{2}-F_{L} \propto \sigma_{T}$. Here $F_{L}$ denotes the longitudinal structure function which is related to the ratio $R=F_{L} /\left(F_{2}-F_{L}\right)$ and $Y_{+}=1+(1-y)^{2}$. The H1 data, taken in 1996 and 1997, have statistical errors of typically $1 \%$ and systematic errors of $2-3 \%$, apart from edges of the acceptance region. In order to reach this precision HERA has been anually increasing the luminosity. The H1 experiment was subject to a major upgrade of its backward apparatus replacing a $\mathrm{Pb}$-Scintillator calorimeter by a Pb-fibre calorimeter of higher granularity, an MWPC by a planar drift chamber and adding a high resolution Silicon strip detector telescope for electron track identification and kinematic reconstruction. This upgrade permitted the measurement to be extended to high $y \leq 0.89$ in order to access $F_{L}$ (Section 2.4) and to low $y \geq 0.003$ in order to reach the $x$ range covered by DIS fixed target experiments. Comparing the data shown in Fig. 5 with the initial HERA data, Fig. 2, one recognizes the impressive progress made in a few years. The data are well described by NLO QCD as discussed in Section 4.2. Consistent results on preliminary $F_{2}$ data were previously obtained by the $\mathrm{H} 1$ and ZEUS Collaborations [35].

The $\mathrm{H} 1$ data help resolving a long standing controversy between NMC and E665 $\mu p$ data and the CCFR $\nu N$ data on the structure function $F_{2}$. As shown in Fig. 6 the H1 data overlap and extrapolate well to the $\mu p$ data. The CCFR $F_{2}$ determination which is being redone [36] was recently criticized regarding the treatment of charm and shadowing [37. Since $F_{2}$ and $x F_{3}$ add up to the measured cross section, an $F_{2}$ reanalysis may affect also the value of $\alpha_{s}$ derived from $x F_{3}$. The CCFR cross section measurement improved in a consistent way the CDHSW cross section data. Those seem not to be in contradiction with muon data [38].

Precision measurements at HERA are essential for calculating the expected rates at LHC energies and also permit to estimate the neutrino scattering cross sections in active galactic nuclei or gamma ray bursts at ultra high energies, up to $E_{\nu} \simeq 10^{12} \mathrm{GeV}$. 
H1 96-97

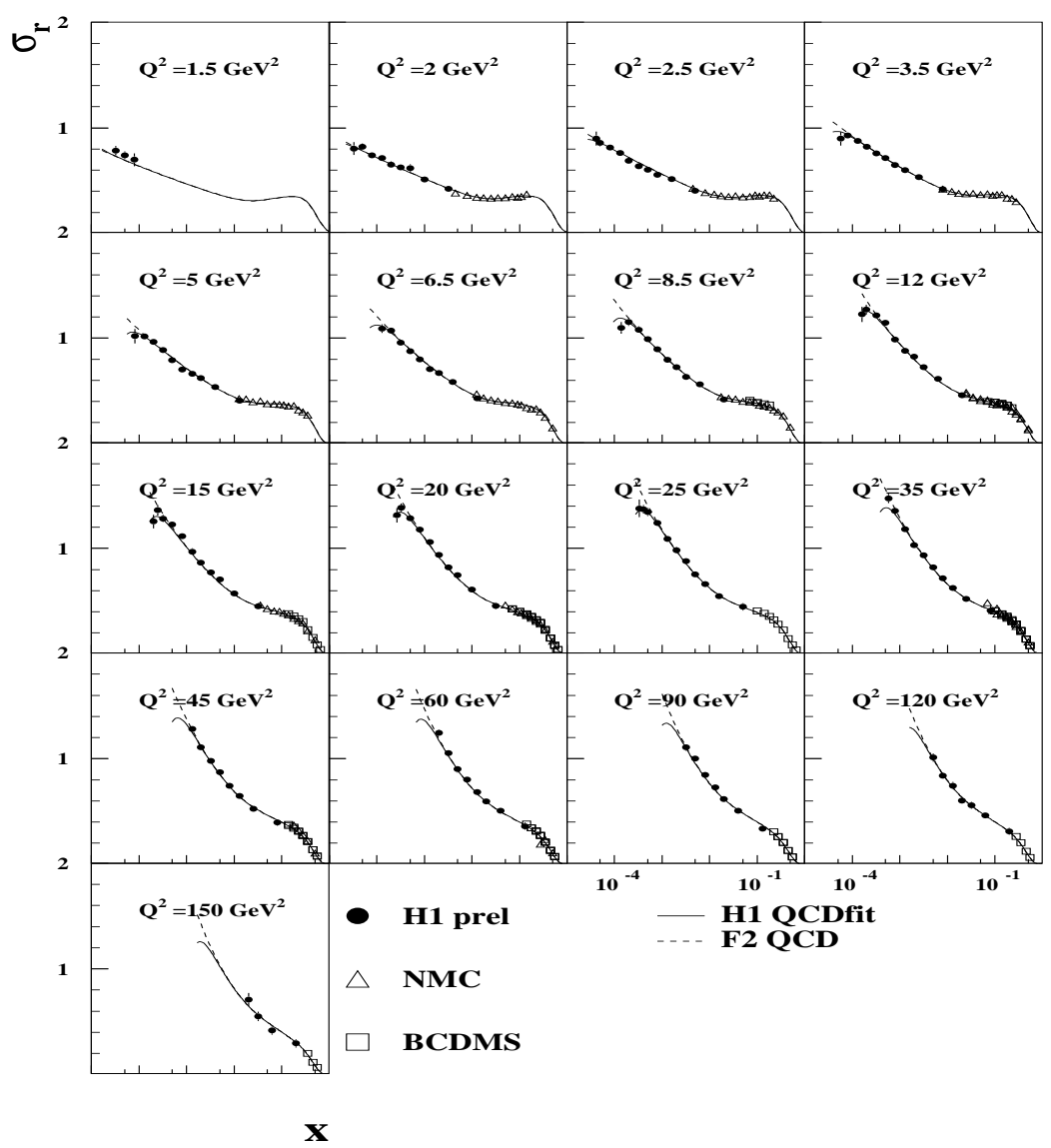

Figure 5: Measurements of the DIS cross section by $\mathrm{H} 1$ compared with NMC and BCDMS $\mu p$ data. The solid curve denotes the fitted cross section in NLO QCD using $\mathrm{H} 1$ and NMC data for $Q^{2} \geq 3.5 \mathrm{GeV}^{2}$. The dashed curve is the structure function $F_{2}$ obtained in the QCD fit which at low $x$ departs from $\sigma_{r}$.

Recently very high energy rates were calculated using the DGLAP equations [39], the GRV approach in DGLAP QCD [40] and a combination of DGLAP and BFKL dynamics [41] which agree remarkably well.

\subsection{Longitudinal Structure Function $\boldsymbol{F}_{L}$}

In the naive QPM the longitudinal structure function $F_{L}$ is zero since partons have spin 1/2. In QCD it acquires a possibly large value due to gluon emission and represents together with $F_{2}$ a strong constraint to the theory in NLO.

The sum of $\nu$ and $\bar{\nu}$ nucleon scattering cross sections is proportional to $2 x F_{1}(1+$ $\epsilon R)-Y_{-} \cdot \Delta x F_{3} / 2 Y_{+}$and thus is sensitive to $R$ where $\epsilon=2(1-y) / Y_{+}$is the polarization 


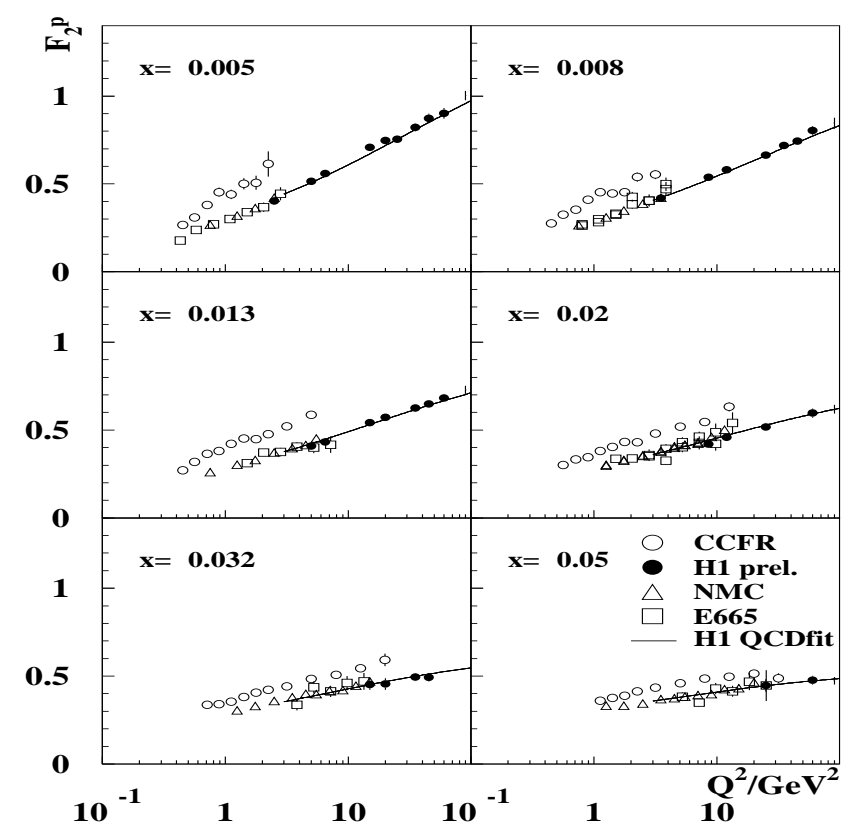

Figure 6: $F_{2}$ structure function data of CCFR, NMC, E665 and H1. The CCFR data were corrected for nuclear effects and for the difference of $F_{2}$ in charged lepton and neutrino scattering [36]. The CCFR data are shown with statistical errors only.

of the $W$ boson exchanged and $Y_{ \pm}=1 \pm(1-y)^{2}$. The CCFR Collaboration has studied the $Q^{2}$ dependence of $R$ for $0.015 \leq x \leq 0.5$ and $Q^{2}<5 \mathrm{GeV}^{2}$ using phenomenological descriptions for the strange and charm quark distribution difference determined by $\Delta x F_{3} \simeq 4 x(s-c)$. The ratio $R$ tends to be large, $R \geq 0.5$, at small $Q^{2} \simeq 1-2 \mathrm{GeV}^{2}$ and $x<0.1$. For $Q^{2}>10 \mathrm{GeV}^{2}$ the function $\Delta x F_{3}=x F_{3}^{\nu}-x F_{3}^{\bar{\nu}}$ was extracted which is of interest for the treatment of massive charm [42].

Using unpolarized targets the HERMES Collaboration measured the ratio of nitrogen to deuterium electroproduction cross sections to be astonishingly small at low $Q^{2}$ [43]. This effect has been attributed to a very large ratio $R_{N} / R_{D} \geq 5$ in the region $0.01<x \leq 0.06$ and $0.5 \leq Q^{2}<1.5 \mathrm{GeV}^{2}$ with as yet unexplained origin.

The measurements of the longitudinal structure function in ep and $\mu p$ scattering are summarized in Fig. 7. The $\mathrm{H} 1$ data were obtained using assumptions for the behaviour of $F_{2}$ in QCD (for $Q^{2}>10 \mathrm{GeV}^{2}$ ) and, independently of QCD, for the derivative $\partial F_{2} / \partial \ln y$ (for $Q^{2}<10 \mathrm{GeV}^{2}$ ) in the high $y$ region [44] where the cross section approaches $F_{2}-F_{L}$. Contrary to fixed target experiments such assumptions are possible since HERA covers more than two orders of magnitude in $y$ where $F_{2}$ can be fixed independently of $F_{L}$. The overall behaviour of $F_{L}$ as a function of $x$ is well described by a QCD fit in NLO using $F_{2}$ data only, i.e. by deriving the gluon (and parton) distributions from scaling violations and then calculating $F_{L}$ (Fig. 7). 


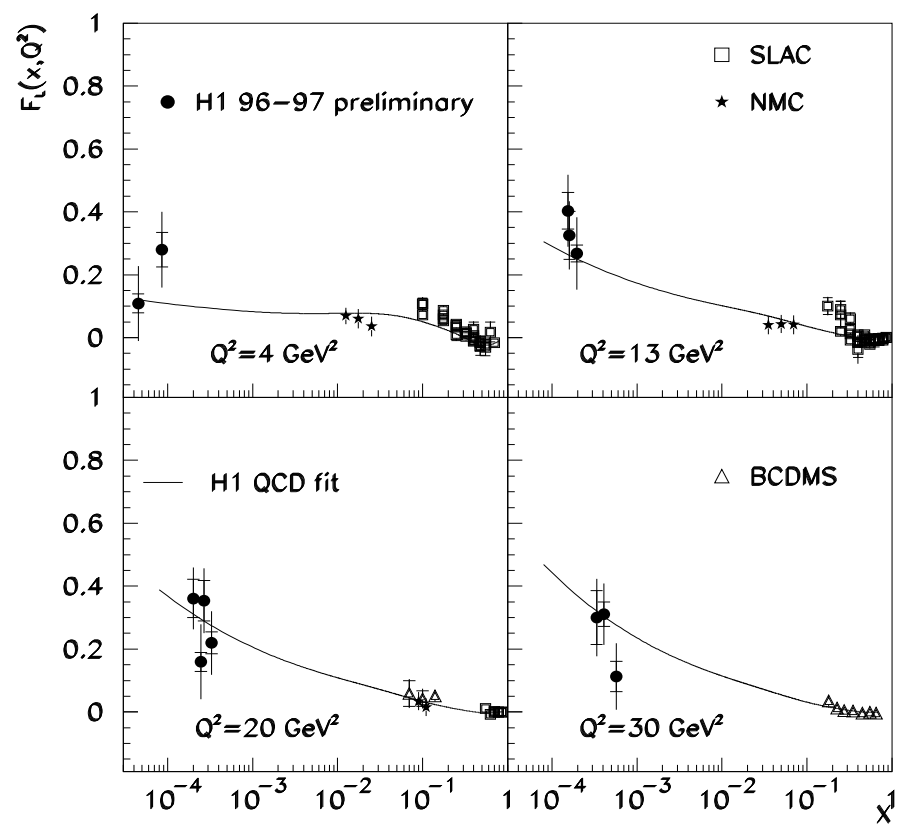

Figure 7: Measurements of the longitudinal structure function $F_{L}\left(x, Q^{2}\right)$ in $e p$ and $\mu p$ scattering. At low $x F_{L}$ is large because of dominant contributions of a large gluon momentum density. The four bins comprise data between $Q^{2}=2,5,15,25$ and $50 \mathrm{GeV}^{2}$, respectively. The curves represent the $\mathrm{H} 1$ calculation of $F_{L}$.

The behaviour of $R$ observed at low $Q^{2} \simeq 1 \mathrm{GeV}^{2}$ and the so far limited accuracy of the $\mathrm{H} 1 F_{L}$ data, obtained with $6.8 \mathrm{pb}^{-1}$, represent a challenge for forthcoming experiments and their theoretical interpretation. This comprises the hypothesis of particularly large higher twist effects and large higher order corrections which at low $x$ and $Q^{2}$ may become even negative in NLO due to a large negative contribution of the gluonic coefficient function [45].

\subsection{Weak Neutral Currents at HERA}

At high $Q^{2} \simeq M_{W, Z}^{2}$ photon, $Z$-boson and $W$-boson exchange are of comparable strength. Thus electroweak interactions can be used to probe proton structure in neutral (NC) and charged current (CC) scattering at HERA in the same experiments. This is demonstrated with the $Q^{2}$ distributions in electron and positron proton NC and $\mathrm{CC}$ scattering, Fig. 8, measured by $\mathrm{H1}\left(e^{+} \mathrm{NC}, \mathrm{CC}\right.$ [46]; $e^{-} \mathrm{NC}, \mathrm{CC}$ [47]) and by ZEUS ( $e^{+} \mathrm{NC}$ [48], $e^{+} \mathrm{CC}\left[49\right.$ and $e^{-} \mathrm{NC}, \mathrm{CC}$ [50]).

The double-differential NC cross section, neglecting the three longitudinal structure functions, is given by two generalized structure functions $\mathbf{F}_{\mathbf{2}}$ and $\mathbf{x F}_{\mathbf{3}}$

$$
\frac{d^{2} \sigma^{ \pm}}{d Q^{2} d x}=\sigma^{ \pm}=\frac{2 \pi \alpha^{2}}{Q^{4} x} \cdot\left[Y_{+} \mathbf{F}_{\mathbf{2}}^{ \pm}+Y_{-} \mathbf{x F}_{\mathbf{3}}^{ \pm}\right] .
$$



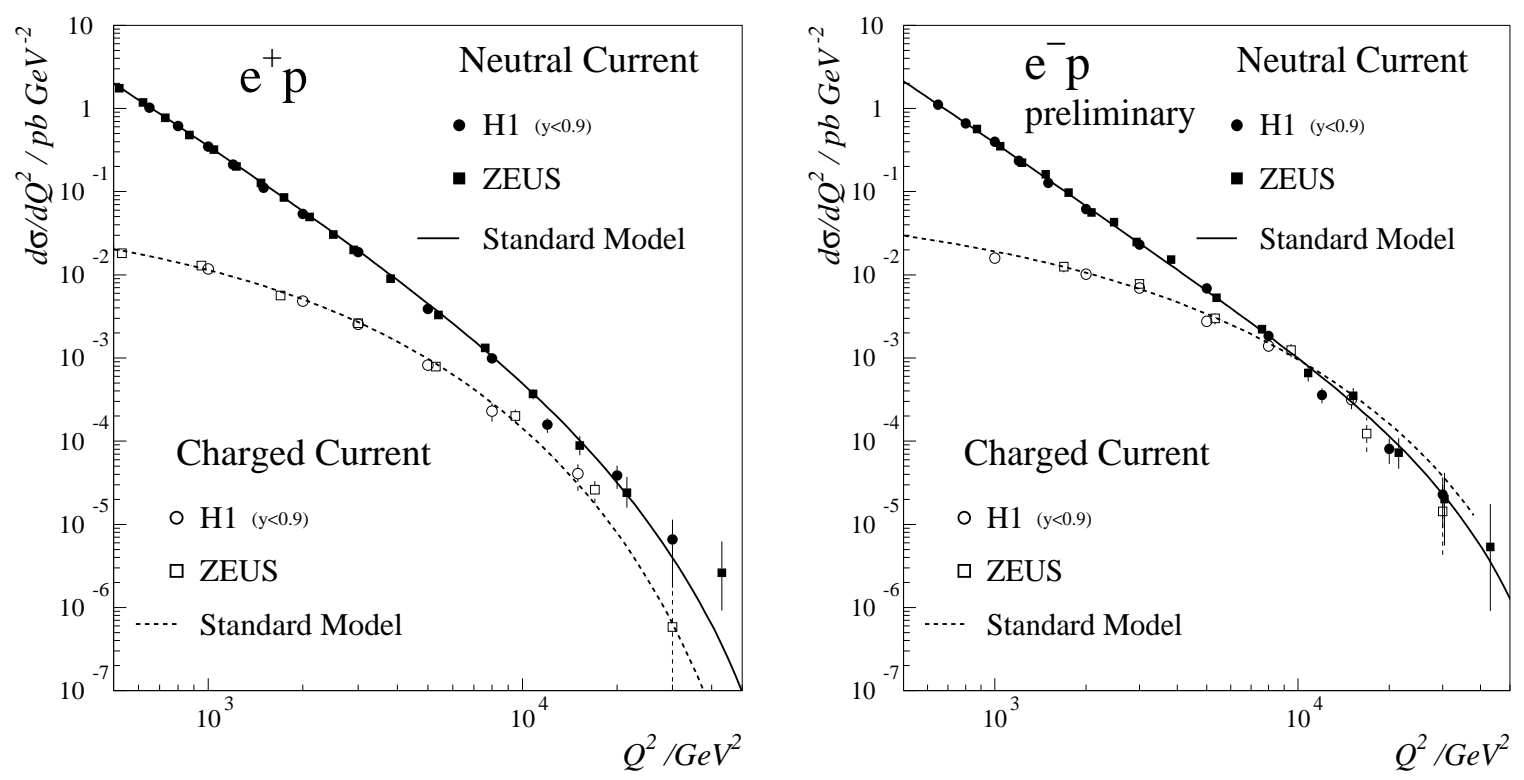

Figure 8: Measurements of the $Q^{2}$ dependence of the positron and the electron proton neutral and charged current scattering cross sections at HERA, using data taken in 1994-1997 $\left(e^{+}, E_{p}=820 \mathrm{GeV}\right)$ and in 1998-1999 $\left(e^{-}, E_{p}=920 \mathrm{GeV}\right)$. Electromagnetic and weak interaction cross sections become of similar strength for $Q^{2} \simeq M_{Z}^{2}, M_{W}^{2}$.

These depend on the quark couplings and distributions but, contrary to hadronic tensor definitions of structure functions [10], they depend also on the weak electron couplings $v, a$ to the $Z$ boson, on the longitudinal electron beam polarization $(\lambda)$ and on the propagators via $\kappa=Q^{2} /\left[4 \sin ^{2} \theta_{W} \cos ^{2} \theta_{W}\left(Q^{2}+M_{Z}^{2}\right)\right]$ where $\theta_{W}$ is the electroweak mixing angle. They comprise five genuine structure functions [11]

$$
\begin{aligned}
\mathbf{F}_{\mathbf{2}}^{ \pm} & =F_{2}+\kappa(-v \mp \lambda a) G_{2}+\kappa^{2}\left(v^{2}+a^{2} \pm 2 \lambda a v\right) H_{2} \\
\mathbf{x F}_{\mathbf{3}}^{ \pm} & =\kappa(\lambda v \pm a) x G_{3}+\kappa^{2}\left(-\lambda\left(v^{2}+a^{2}\right) \mp 2 a v\right) x H_{3},
\end{aligned}
$$

defined in Section 1, Eqs. 目 and 2. The $\mathbf{x F}_{\mathbf{3}}$ term $\left(\propto Y_{-}\right)$contributes sizeably only at large $y$ and high $Q^{2}$. The high $Q^{2} \mathrm{NC}$ cross sections measured currently at HERA for $\lambda=0$ are approximately given by

$$
\sigma^{ \pm} \simeq Y_{+} \cdot F_{2} \pm \kappa a Y_{-} \cdot x G_{3} .
$$

This causes a positive charge asymmetry between electron and positron scattering which is proportional to $a a_{q}$, i.e. parity conserving, and which is determined by the function $x G_{3}$ measured previously by BCDMS at lower $Q^{2}$ for an isoscalar target, see Fig. 囵.

The H1 Collaboration has performed measurements of double differential NC scattering cross sections [47] using $35.6 \mathrm{pb}^{-1}$ of $e^{+}$data [46] taken in 1994-97 at 
$E_{p}=820 \mathrm{GeV}$ and $15 \mathrm{pb}^{-1}$ of $e^{-}$data 47 taken in 1998-99 at $E_{p}=920 \mathrm{GeV}$. A comparison of the cross section measurements with electrons and positrons is illustrated in Fig. 9 which agrees with expectation based on the $\gamma Z$ interference in $\mathrm{NC}$ scattering.

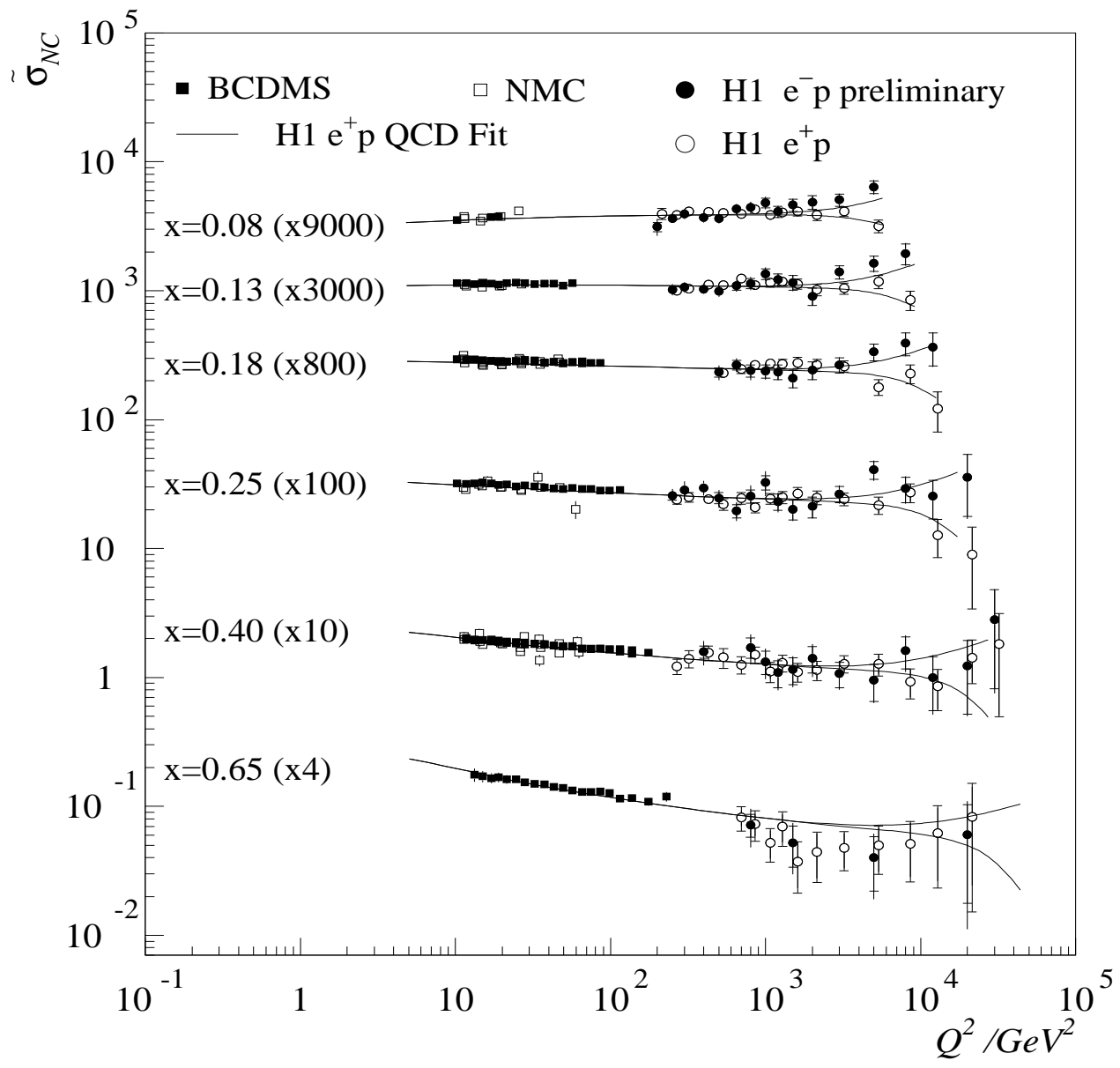

Figure 9: Measurements of the double differential $\mathrm{NC} e^{+}$and $e^{-}$proton scattering cross sections by $\mathrm{H} 1$ and NMC and BCDMS data. The high $Q^{2} \mathrm{H} 1$ data are well described by a QCD fit, which may even be restricted to $Q^{2}<150 \mathrm{GeV}^{2}$ [47, representing a remarkable confirmation of the DGLAP evolution in $Q^{2}$. A fit to solely $\mathrm{H} 1$ and NMC data passes through the $\mathrm{H} 1$ points but undershoots the BCDMS data at largest $x$ significantly. 


\section{Light and Charm Quark Distributions}

\subsection{Charged Currents and Up and Down Quarks}

New information on the up and down quark distributions became available from improved measurements of the charged current cross section at HERA by H1 and ZEUS. The double-differential CC scattering cross section is given as

$$
\frac{d^{2} \sigma_{c c}^{ \pm}}{d x d y}=\frac{G^{2}}{2 \pi} \cdot\left(\frac{M_{W}^{2}}{Q^{2}+M_{W}^{2}}\right)^{2} \cdot s \frac{1 \pm \lambda}{2} \cdot\left[Y_{+} W_{2}^{ \pm} \mp Y_{-} x W_{3}^{ \pm}\right]
$$

where $G$ is the Fermi constant and $M_{W}$ the mass of the $W$ boson. The CC cross section contains two structure functions for a given lepton beam charge and is proportional to $s$. The HERA energy is equivalent to $53.9 \mathrm{TeV}$ neutrino beam energy in a neutrinonucleon fixed target experiment. The energy dependence is damped for $Q^{2} \geq M_{W}^{2}$. In the QPM the CC structure functions are combinations of up and down quark distribution sums, i.e. $W_{2}^{+}=D+\bar{U}, W_{2}^{-}=U+\bar{D}, x W_{3}^{+}=D-\bar{U}$ and $x W_{3}^{-}=U-\bar{D}$ with $U=x(u+c)$ and $D=x(d+s)$. At large $x \geq 0.3$ the valence quark distributions $u_{v}$ and $d_{v}$ dominate the interaction cross sections, i.e.

$$
\begin{array}{rlr}
\sigma\left(e^{+} p \rightarrow \bar{\nu} X\right) & \propto \bar{U}+(1-y)^{2} D \rightarrow(1-u)^{2} x d_{v} \\
\sigma\left(e^{-} p \rightarrow \nu X\right) & \propto U+(1-y)^{2} \bar{D} \rightarrow \quad x u_{v}
\end{array}
$$

for $x \rightarrow 1$. A complete set of double differential $e^{ \pm} p$ CC cross section data was presented by H1 using $36 \mathrm{pb}^{-1}$ of positron-proton data (1994-1997) [46] and $15 \mathrm{pb}^{-1}$ of electron data (1998-1999) [47]. The $U$ dominated $e^{-} p$ cross section was found to be about 5 times larger than the $e^{+} p$ cross section at $Q^{2} \simeq 10,000 \mathrm{GeV}^{2}$. The $e^{+} p$ CC data of $\mathrm{H} 1$ are consistent with the published measurement of the ZEUS Collaboration 49 based on $47.7 \mathrm{pb}^{-1}$, Fig. 10. The NC and CC measurements at high $Q^{2}$ are of particular interest for the determination of the $d / u$ ratio at high $x$ because their interpretation is free of nuclear corrections. Yet, an order of magnitude increase in luminosity is still required to access the high $x$ region which represents one of the goals of the HERA luminosity upgrade programme.

Deuterium binding corrections were recently reconsidered, and $d_{v}$ was adjusted to be larger than previously assumed [51], the ratio $d_{v} / u_{v}$ for $x \rightarrow 1$ tending to 0.2 . An enlarged $d$ quark distribution fits to the $W^{ \pm}$charge asymmetry data in $p \bar{p}$ collisons. Violation of $u$ and $d$ quark symmetry in protons and neutrons, however, which was suggested to explain the difference between the CCFR and NMC $F_{2}$ data [52], leads to too large a $W$ asymmetry [53]. 
ZEUS CC 1994-97

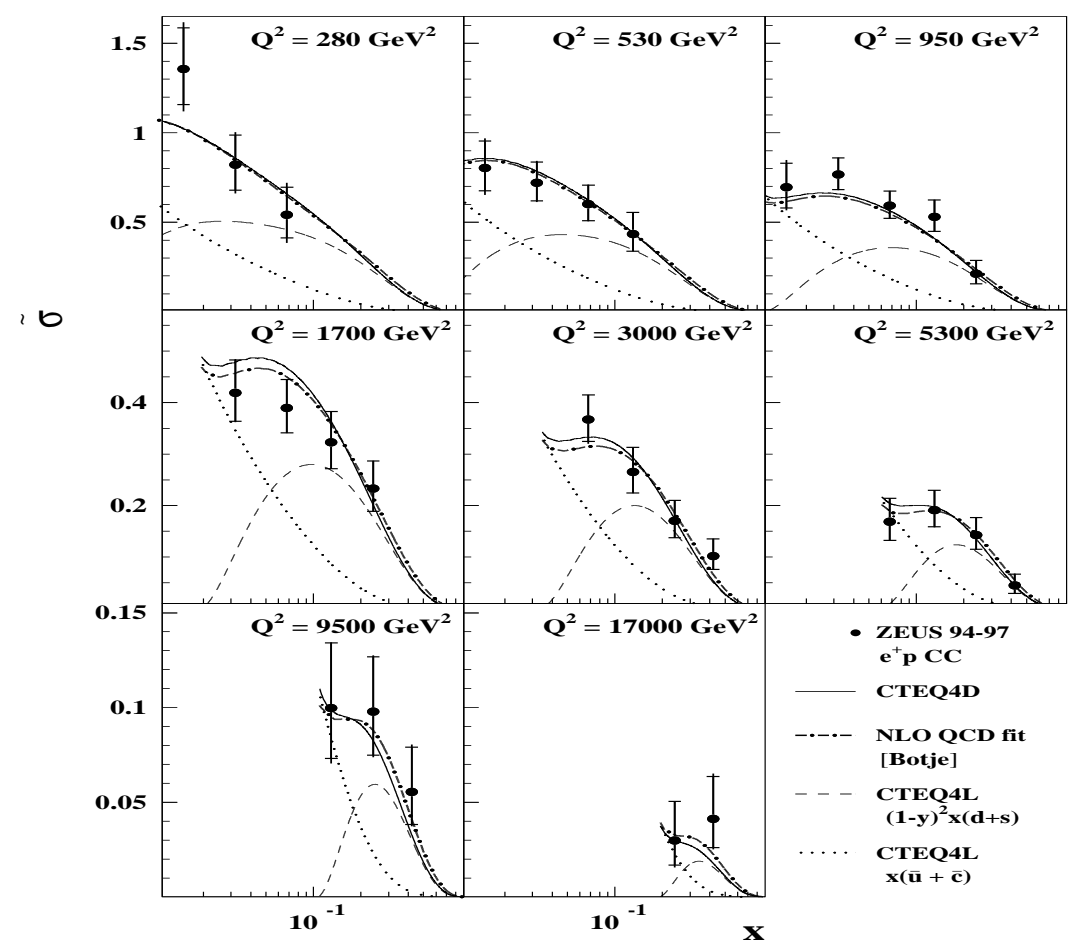

Figure 10: ZEUS measurement of the $\mathrm{CC} e^{+} p$ scattering cross section compared with $\bar{U}$ and $D$ quark distributions, see Eq. 10, and different QCD fits.

\subsection{Sea Quarks}

Interesting data become available on the flavour asymmetry in the nucleon sea. From a high statistics measurement of Drell-Yan muon pair production in $p p$ and $p d$ collisions at the Tevatron, the E866/NuSea Collaboration obtained for $\int_{0}^{1}(\bar{u}-\bar{d}) d x$ a value of $-0.118 \pm 0.011$ at $\left\langle Q^{2}\right\rangle=54 \mathrm{GeV}^{2}$ [54]. This confirms and also significantly improves the previous NMC result of $-0.15 \pm 0.04$ which was derived from a measurement of the Gottfried sum rule $\int_{0}^{1}\left[\left(F_{2}^{p}-F_{2}^{n}\right) / x\right] d x=1 / 3+2 / 3 \cdot \int_{0}^{1}(\bar{u}-\bar{d}) d x$. The measured ratio $\bar{d} / \bar{u}$ as a function of $x$ is shown in Fig. 11. The data have considerable impact on global parametrizations of parton distributions. A consistent result, albeit of less statistical accuracy, was obtained by the HERMES Collaboration [55] with a measurement of semi-inclusive $\pi^{ \pm}$production in unpolarized ep and ed scattering at lower $\left\langle Q^{2}\right\rangle=2.3 \mathrm{GeV}^{2}$. A violation of flavour symmetry is not predicted in perturbative QCD which points to non-perturbative effects such as Pauli blocking and pion clouds. In the latter model the nucleon is expanded in a Fock state of mesons and baryons. Phenomenologically one finds more $\pi^{+}$than $\pi^{-}$in the nucleon with a momentum distribution peaking at $x_{\pi} \simeq 0.2$ 56. 

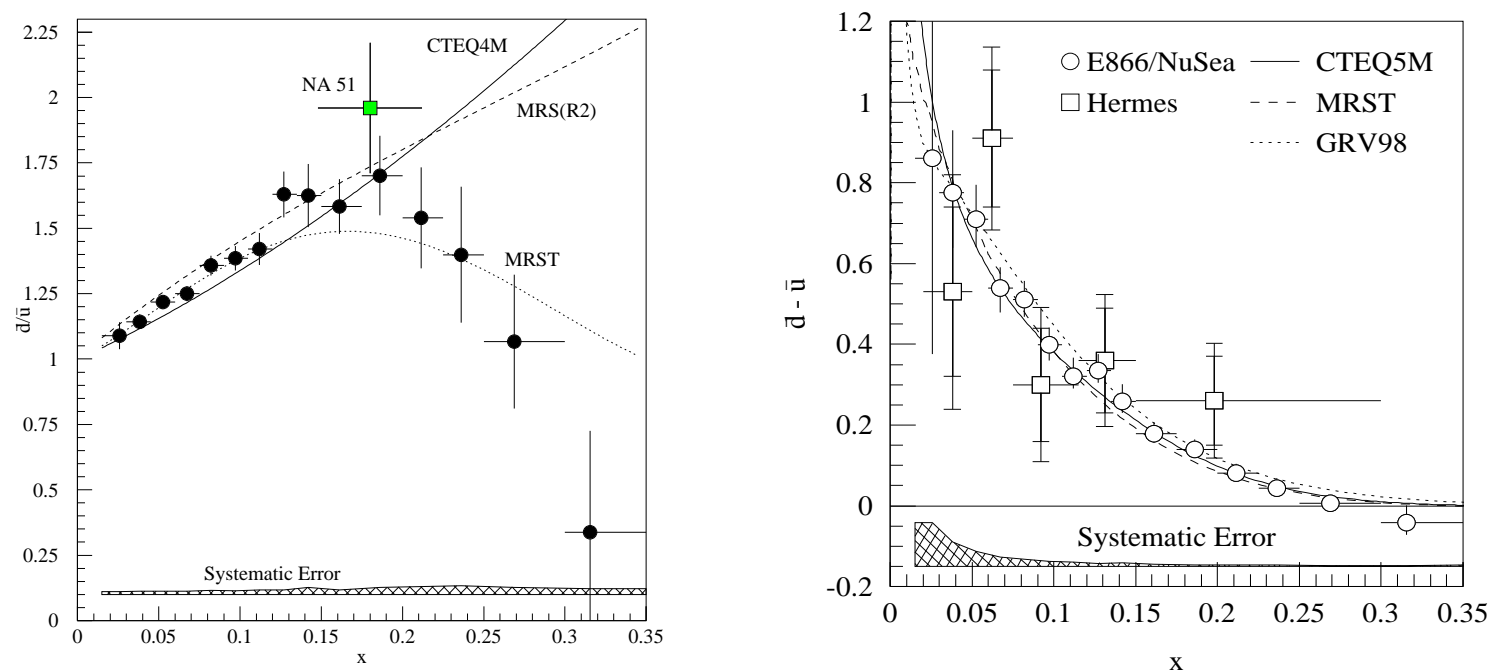

Figure 11: Measurements of $\bar{d} / \bar{u}$ and of $\bar{d}-\bar{u}$ by NA 51, E866/NuSea and by HERMES compared with recent structure function fits.

The NuTeV Collaboration [57] determined the strange quark distribution to be about $1 / 2$ of the averaged nucleon sea, i.e. $s=[0.42 \pm 0.07$ (syst) $\pm 0.06($ stat $)] \cdot(\bar{u}-$ $\bar{d}) / 2$, in agreement with previous analyses of dimuon production in neutrino-nucleon scattering experiments.

Indications for a difference of the strange and anti-strange quark distributions at large $x \simeq 0.6$ were obtained in a recent reanalysis and global fit of DIS and Drell-Yan data [38]. Sensitivity to $(s-\bar{s})$ in this analysis comes from the CDHS data measuring $\sigma^{\nu}-\sigma^{\bar{\nu}} \propto x(s-\bar{s})+Y_{-} x\left(u_{v}+d_{v}\right)$ at high $x$. Such a strange asymmetry is possible in models considering states as $K^{+} \Lambda$ to be intrinsic to the nucleon where $K^{+}$yields $\bar{s} \propto(1-x)$ and $\Lambda$ yields $s \propto(1-x)^{3}$ [58].

\subsection{Charm}

Charm, as was already noticed by Witten in 1976, may "subject non-Abelian theories to a rigorous experimental test by measuring the charmed quark contribution to structure functions" [59]. Since then the charm and beauty treatment in perturbative QCD has been worked out to higher orders [60]. Variable flavour schemes are being studied [61] to correctly handle the heavy flavour contributions near and beyond threshold in analyses of parton distributions, of the gluon distribution and of $\alpha_{s}$. A new measurement of the charm structure function $F_{2}^{c \bar{c}}$ was performed by the ZEUS Collaboration [62] using the $\Delta M$ tagging technique for $D^{*} \rightarrow K 2 \pi$ and $K 4 \pi$, Fig. 12 . The relative contribution of charm is large, reaching $30 \%$ at low $x<0.001$ for $Q^{2} \simeq$ $100 \mathrm{GeV}^{2}$. This large fraction is due to photon-gluon fusion as the dominant process 

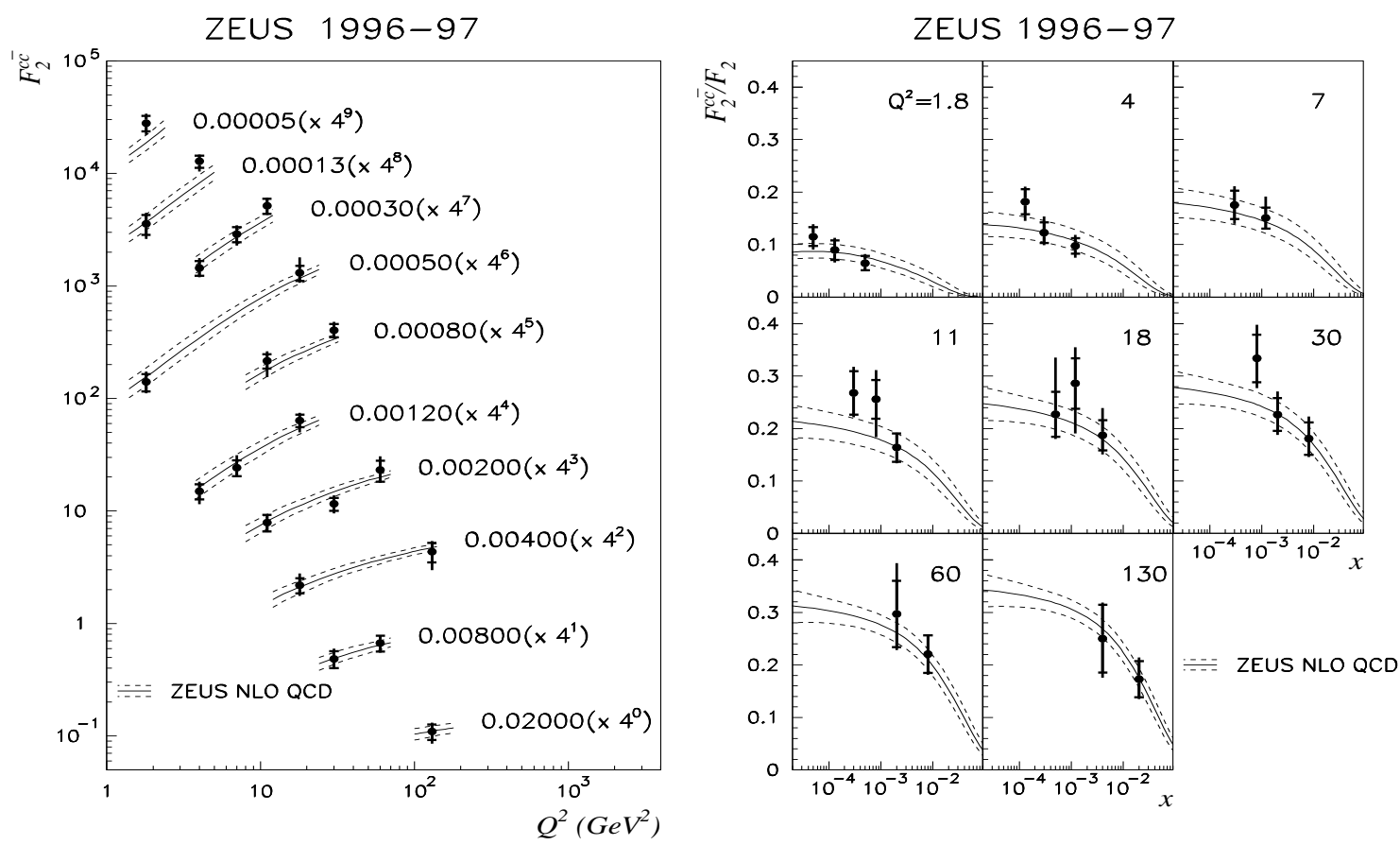

Figure 12: Measurement of $F_{2}^{c \bar{c}}\left(x, Q^{2}\right)$ and of the ratio $F_{2}^{c \bar{c}} / F_{2}$ in $e p$ scattering at HERA by the ZEUS Collaboration with $37 \mathrm{pb}^{-1}$ of data. The dashed error bands denote the uncertainty of the QCD fit which is dominated by the charm quark mass range chosen to be 1.2 to $1.6 \mathrm{GeV}$.

for charm production. Further experimental progress at HERA towards high precision will be achieved with new or upgraded Silicon vertex detectors, higher luminosity, inclusion of further final states and dedicated track triggers.

\section{Gluon Distribution and Coupling Constant $\boldsymbol{\alpha}_{s}$}

\subsection{Scaling Violations at Low $x$}

Scaling violations in the DIS $Q^{2}$ region down to low $x \simeq 0.00005$ can be successfully described in the DGLAP formalism. This is again demonstrated with the new precise cross section measurement of H1, Fig. 5. Conventional QCD fits use parametrizations of parton distributions at a starting scale $Q_{o}^{2}$ and evolve them in $Q^{2}$ to highest $Q^{2} \geq$ $M_{Z}^{2}$ values up to order $\alpha_{s}^{2}$. However, the splitting functions have expansions which contain also powers of $\ln (1 / x)$. These are large at low $x$, such that $\alpha_{s} \ln (1 / x) \simeq 1$, and yet do not seem necessary to phenomenologically describe the observed structure function behaviour. Calculations are performed in order to account for these $\ln (1 / x)$ 
terms [20] and to cure perhaps the instability of the BFKL equation in NLO 63]. Indications were reported for the presence of $\ln (1 / x)$ terms in inclusive DIS data 64. Experimentally even higher precision is both required and possible for the structure function measurements, including $F_{L}$, which may lead to crucial tests of QCD at low $x$. Due to unitarity constraints one expects to find saturation of the rising behaviour of $F_{2}$ which, however, seems to be beyond the low $x$ range accessible by HERA in the DIS region.

Scaling violations are conveniently studied using the $\ln Q^{2}$ derivative of $F_{2}$. In Fig. 13 the structure function $F_{2}$ from $\mathrm{H} 1$ is shown as a function of $Q^{2}$ for $x<$ 0.01. The $\ln Q^{2}$ dependence is non-linear and can be well described by a quadratic expression $P_{2}=a+b \ln Q^{2}+c\left(\ln Q^{2}\right)^{2}$ (solid lines) which nearly coincides with the NLO QCD fit (dashed lines). The local derivatives $\partial F_{2} / \partial \ln Q^{2}$ determined from the new $\mathrm{H} 1 F_{2}$ data are not constant in $Q^{2}$ and also depend on $x$. Approximately they can be described for each bin of $x$ by $b+2 \cdot c \ln Q^{2}$. Small deviations from this behaviour occur in NLO QCD. Using this expression the derivatives are determined at fixed $Q^{2}$ and displayed as functions of $x$ in Fig. 13. There is no departure observed from a rising behaviour of the $\ln Q^{2}$ derivatives down to $Q^{2}=3 \mathrm{GeV}^{2}$. If such a plot is made as a one-dimensional distribution, using the derivatives calculated for each bin of $x$ at the mean $Q^{2}$ of a given bin, then the derivative $d F_{2} / d \ln Q^{2}$ flattens starting at $Q^{2} \simeq 6 \mathrm{GeV}^{2}$ [65]. In the region covered by the $\mathrm{H} 1$ data this behaviour reflects the restriction of the kinematic range of the measurement. Some analyses of the ZEUS data extending to lower $Q^{2} \simeq 1 \mathrm{GeV}^{2}$ introduce screening corrections in order to describe the behaviour of $F_{2}$ 66, 67]. Both $F_{2}$ and $F_{L}$ in this region should be measured with still higher accuracy (see Section 2.1.) as these permit important information to be deduced on the dynamic interplay of gluon and sea distributions, on the effect of higher order and power corrections and on the shadowing phenomenon.

\subsection{Gluon Distributions}

In QCD the $Q^{2}$ evolution of $F_{2}$ is governed by the strong interaction coupling constant $\alpha_{s}$. The evolution relates the quark distributions to the gluon distribution $x g$. The H1 Collaboration has performed a new NLO QCD fit to the H1 and NMC inclusive crosssection data. It uses the DGLAP evolution equations for three light flavours with the charm and beauty contributions added according to the NLO calculation of the bosongluon fusion process 68. The proton structure function $F_{2}$ is a superposition of two independent functions with different evolutions, i.e. $F_{2}=5 / 18 \cdot S+1 / 6 \cdot N$, where the singlet function $S=U+D$ is the sum of up and down quark distributions and the non-singlet function $N=U-D$ is their difference. In the new $\mathrm{H} 1$ fit a different linear combination is introduced such that $U=2 / 3 \cdot V+A$ and $D=1 / 3 \cdot V+A$. In a simplified parton model ansatz with $\bar{u}=\bar{d}$ and $s+\bar{s}=(\bar{u}+\bar{d}) / 2$ one finds $V=3 / 4 \cdot\left(3 u_{v}-2 d_{v}\right)$. This allows the quark counting rule to be applied which 

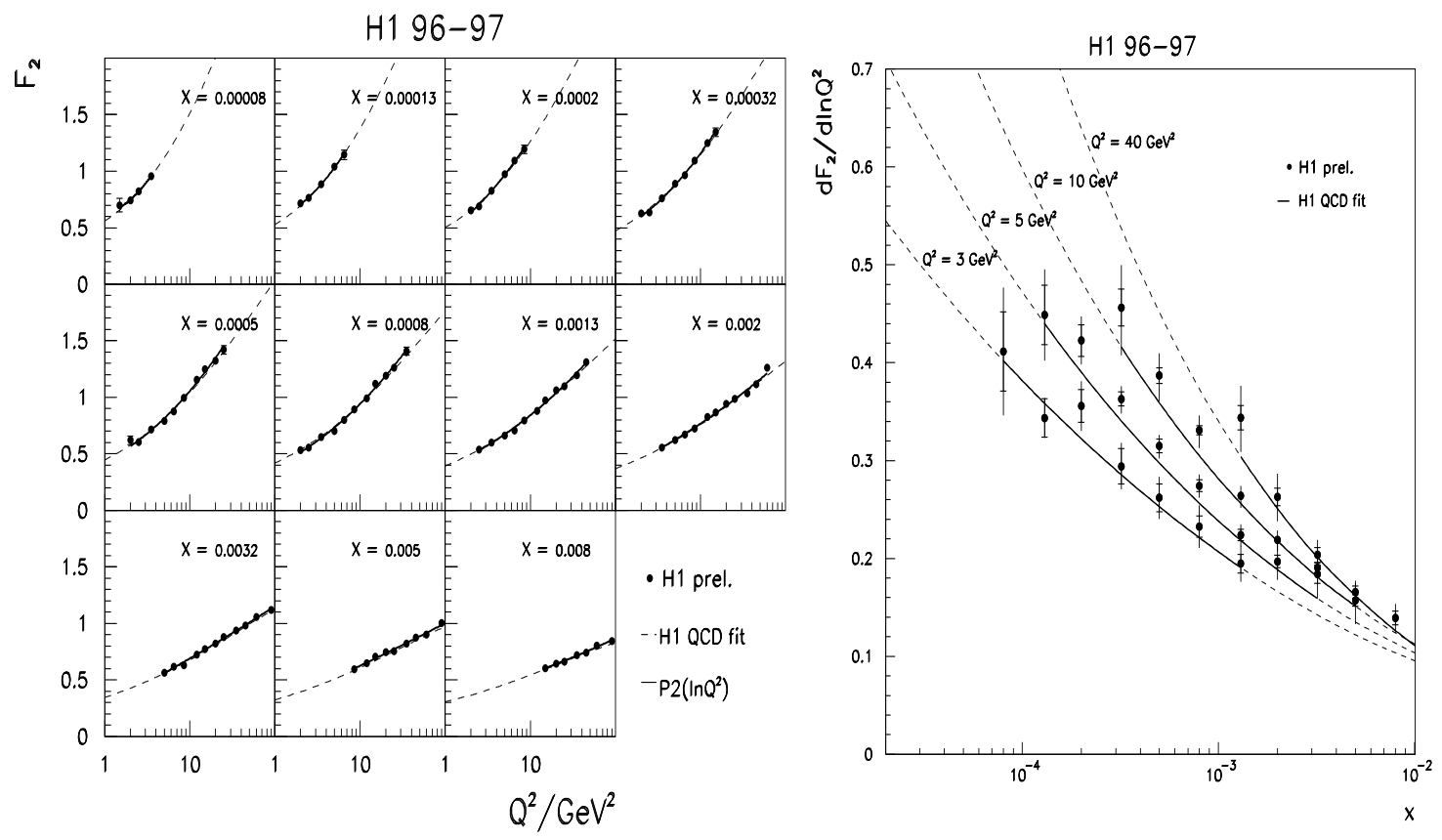

Figure 13: New preliminary $\mathrm{H} 1$ data show $F_{2}\left(x, Q^{2}\right)$ to be non-linear in $\ln Q^{2}$ at low $x$ (left). The derivative $\partial F_{2} / \partial \ln Q^{2}$ is a continuously falling function of $x$ for $Q^{2} \geq 3 \mathrm{GeV}^{2}$ (right).

constrains $\int V d x=3$. This ansatz is used to fit the cross-section data, Fig. 5, for $3.5 \leq$ $Q^{2} \leq 3000 \mathrm{GeV}^{2}$ assuming $\alpha_{s}\left(M_{Z}^{2}\right)=0.118$. It is written in the $\overline{M S}$ renormalization scheme and generalized to account for the measured difference $\bar{u}-\bar{d}$ and the fraction of strange quarks, see Section 3. The salient feature of this new analysis is that it applies to DIS proton data only but correctly determines the gluon momentum fraction to be about 0.45 at $Q^{2}=10 \mathrm{GeV}^{2}$. The gluon distribution resulting from this fit is shown in Fig. 14 (left). The inner error band defines the experimental uncertainty of a few per cent at low $x$ using the treatment of correlated systematic errors of [69]. The outer error band comprises uncertainties due to dependencies on the fit parameters $\left(Q_{m i n}^{2}, Q_{o}^{2}, \alpha_{s}, m_{c}\right)$ and on the choice of parametrizations for the initial distributions. A remarkable feature of $x g$ is the crossing point at $x \simeq 0.06$ which is analogous to the Bjorken scaling behaviour of $F_{2}$ and reflects the conservation of the gluon and quark momenta. In Fig. 14 (right) the gluon distribution is seen to agree very well with $x g$ unfolded from the charm structure function DIS and photoproduction data of H1 [70] which confirms hard scattering factorization. It has early been recognized that in photoproduction $\left(Q^{2} \simeq 0\right)$ the charm mass provides a hard scale [71].

While $x g$ at low $x$ is well determined by the HERA structure function measurements, there are sizeable uncertainties of one order of magnitude at high $x \simeq 0.6[72]$. 

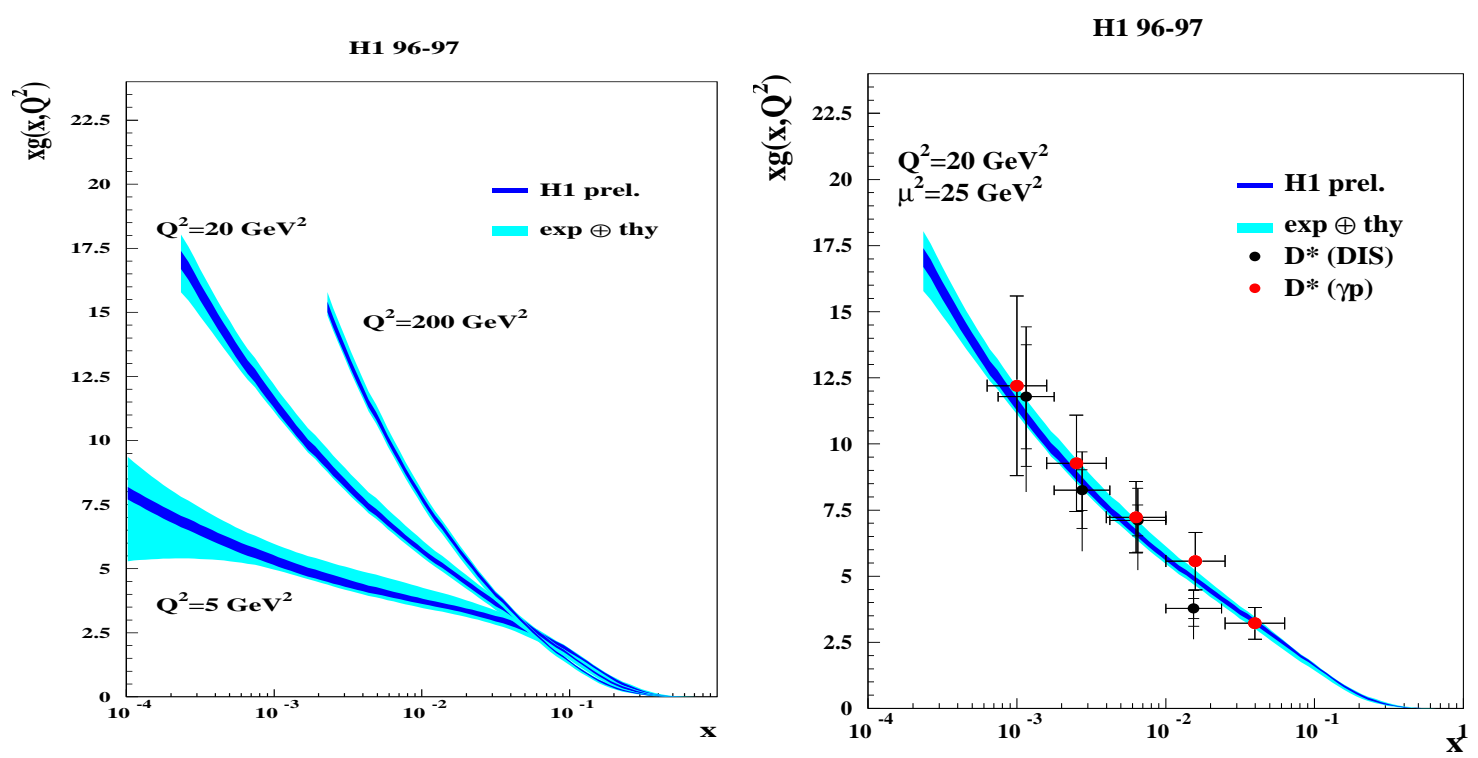

Figure 14: Determination of $x g$ by H1 using NMC and H1 $l p$ data in NLO QCD (left). Comparison of $x g$ from scaling violations with the unfolded gluon distribution from charm $D^{*}$ measurements by H1(right).

The gluon distribution is accessed at high $x$ by quark-gluon Compton scattering leading to direct photon emission [73]. In a recent experiment by the E706 Collaboration [74 the photon $p_{T}$ spectrum was found to exceed QCD expectation by a factor of about two which has been phenomenologically cured by a Gaussian transverse momentum smearing with $k_{T}$ of $1 \mathrm{GeV}$, larger than the intrinsic $k_{T}$ value of about $0.4 \mathrm{GeV}$ [72]. High $E_{T}$ jet data at large rapidities are sensitive also to $x g$ at large $x$ and lead to a rather high gluon distribution. Inclusion of different data sets yields remarkably differing results. Resolving the issue of $x g$ at high $x$ is essential for a reliable prediction of Higgs production in $p p$ colliders. It is necessary since the high $x$ exponent $c_{g}$ of $x g \propto(1-x)^{c_{g}}$ is known to be correlated with $\alpha_{s}$. In this respect precision measurements of structure functions at high $x$ are important. Since $F_{2}$ vanishes as $(1-x)^{3}$, any measurement error at large $x$ is amplified like $1 /(1-x)$. The HERA collider experiments with their unique possibility to overconstrain the kinematics can be expected to lead to precision data also at high $x$ [75] when the luminosity is upgraded.

Recently updates of the GRV parametrizations were presented [76]. New sets of fits were made by the MRST [77] and the CTEQ groups [78]. GRV98 uses DIS, $n / p$ and Drell-Yan data assuming $\alpha_{s}\left(M_{Z}^{2}\right)=0.114$. MRST99 uses direct photon data of the WA70 experiment for different $k_{T}$ and varies the $d / u$ ratio, $\alpha_{s}$ and $m_{c}$. CTEQ5 does not use direct photon data but analyzes high $E_{T}$ jet data instead. Sets are pro- 
vided for different renormalization schemes and heavy quark treatments. As a consequence there exists a variety of parametrizations illustrating the still large flexibility of theoretical assumptions and pointing to possible experimental contradictions. An interesting attempt was made recently [79] to quantify the experimental uncertainties of parton distributions resulting from global QCD fits to DIS data.

\subsection{Determinations of $\alpha_{s}$}

New determinations of $\alpha_{s}\left(M_{Z}^{2}\right)$ with structure function data were presented recently. Conventional analyses parametrize a set of input quark distributions and $x g$ at certain input scale $Q_{o}^{2}$ using the DGLAP equations to NLO to calculate the theoretical expectation. Minimization of a $\chi^{2}$ function determines $\alpha_{s}$ and the roughly 10-15 parton distribution parameters. The treatment of systematic errors affects both the central value and the error size of $\alpha_{s}\left(M_{Z}^{2}\right)$. At low $Q^{2}$ power corrections to the logarithmic evolution may be sizeable and anticorrelate with $\alpha_{s}$. Since analyses differ in these assumptions and use different sets of data, one may not be surprised to still find some spread of the quoted values of $\alpha_{s}\left(M_{Z}^{2}\right)$. Using the SLAC, BCDMS and NMC $p$ and $n$ structure function data and taking into account systematic error correlations and higher twists $\propto 1 / Q^{2}$, a value of $\alpha_{s}\left(M_{Z}^{2}\right)=0.1183 \pm 0.0021($ exp $) \pm 0.0013$ (thy) has been derived [80]. A similar analysis 81] including the published HERA data and adding all errors in quadrature yields $\alpha_{s}\left(M_{Z}^{2}\right)=0.114 \pm 0.002(e x p)_{-0.004}^{+0.006}(t h y)$ which is closer to a previous determination of $\alpha_{s}\left(M_{Z}^{2}\right)$ based on SLAC and BCDMS data [82]. The quoted theoretical errors represent the uncertainties of the renormalization scale $\mu_{r}$, the former analysis compensating part of the $\mu_{r}$ dependence with the higher twist contribution.

The theoretical uncertainties are diminished in NNLO calculations. So far only partial results are available on the 3-loop splitting functions while the $\beta$ function and the coefficient functions are known [83]. This gave rise to a revival of moment analyses. In 84 the $x F_{3}$ data of the CCFR Collaboration are reconstructed using orthogonal Jacobi polynomials. Power corrections are considered and a value of $\alpha_{s}\left(M_{Z}^{2}\right)=0.118 \pm 0.002$ (stat) \pm 0.005 (syst) \pm 0.003 (thy) is obtained in NNLO corresponding to 0.120 in NLO. While this uses a pure non-singlet function, not coupled to the gluon distribution, a new analysis of SLAC, BCDMS, NMC, ZEUS and $\mathrm{H} 1$ data using Bernstein polynomials of $F_{2}$ yields $\alpha_{s}\left(M_{Z}^{2}\right)=0.1163 \pm 0.0023$ in NNLO with a single error supposed to comprise all experimental and theoretical uncertainties. This analysis 850 is extended to a $Q^{2}$ range of 2.5 to $230 \mathrm{GeV}^{2}$ and includes power corrections. Its NLO result is 0.1175 , and moments of $x g$ are determined.

Although all these analyses represent quite remarkable theoretical and experimental progress, one still has to be cautious. The systematic error treatments of these analyses differ. An important issue is the possible incompatibility of different data sets. For example, the combination of SLAC and BCDMS data yields an $\alpha_{s}\left(M_{Z}^{2}\right)$ value 
near to 0.114. Yet, this is known to result from a superposition of the BCDMS data favouring a value of about 0.110 with the SLAC data preferring $\alpha_{s} \simeq 0.120$. Furthermore, the moment analyses, while theoretically advanced to NNLO, shift the data weight to large $x$ where the accuracy of the data is less impressive. Moreover, there is a dependence of the result on the minimum $Q^{2}$ considered [81] which often leads to the introduction of power corrections with phenomenological $x$ dependence. Finally the likely presence of $\ln (1 / x)$ terms will affect the data interpretation. It is thus concluded that the great potential of DIS data to determine $\alpha_{s}\left(M_{Z}^{2}\right)$ requires still much more work in order to determine $\alpha_{s}$ at the one per cent level of accuracy.

Interesting ideas are pursued to replace in the QCD analysis $x g$ by the derivative $\partial F_{2} / \partial \ln Q^{2}$ [81, 86] and to develop the method of truncated moments 87 in order to avoid the low $x$ region in analyses of structure functions other than $F_{2}$. The approach of double asymptotic scaling at low $x$ of $F_{2}$ 15 represents a three parameter solution of the DGLAP equations and may lead to a particularly accurate determination of $\alpha_{s}\left(M_{Z}^{2}\right)$ [88, 89]. This solution predicts a steady increase of $x g$ towards low $x$ which yet has to be damped at certain $x$ and $Q^{2}$ since $x g$ may not exceed the proton size $\pi r_{p}^{2}$ by too big an amount 90 .

\section{On the Future of Deep Inelastic Scattering}

During the year 2000 the HERA luminosity will be upgraded 91 in order to provide an integrated luminosity of $150 \mathrm{pb}^{-1}$ per year. Variations of proton and electron beam energies and the use of electron polarization in colliding mode will further enable the electroweak structure function measurements and enhance the discovery potential of the machine. The modifications of HERA are accompanied by major detector upgrades of the luminosity, forward tracking and Silicon vertex detectors of H1 and ZEUS.

The main injector neutrino oscillation detector at Fermilab $\left(\right.$ MINOS $\left._{\text {near }}\right)$ will lead to precise, high statistics data $\left(\simeq 4 \cdot 10^{7}\right.$ events/year $)$ on the six structure functions $\left(F_{2}\right.$, $x F_{3}$ and $F_{L}$ for $\nu F e$ and $\bar{\nu} F e$ scattering) which is necessary to disentangle the nucleon sea, i.e. to measure $\bar{u}+\bar{d}, c$ and $s$ [92]. Measurements of the nuclear dependence of neutrino DIS cross sections using additional targets will determine $\nu A$ shadowing and perhaps help resolving the CCFR-NMC puzzle, Section 2.2. Increase of neutrino energy by a factor of 10 would be possible in a $250 \mathrm{GeV}$ muon storage ring providing extremely intense neutrino beams [93, 94].

The obvious next step in electron-proton DIS is a new ep machine 95. The proposed linear collider at DESY, TESLA, may provide collisions of electrons of up to about $500 \mathrm{GeV}$ against HERA protons of nearly $1 \mathrm{TeV}$. A similar energy of $\sqrt{s} \simeq 1.5 \mathrm{TeV}$ can be obtained in ep collisions at LEP-LHC energies. These machines differ in technology, luminosity and kinematics. Yet one can envisage extending the 


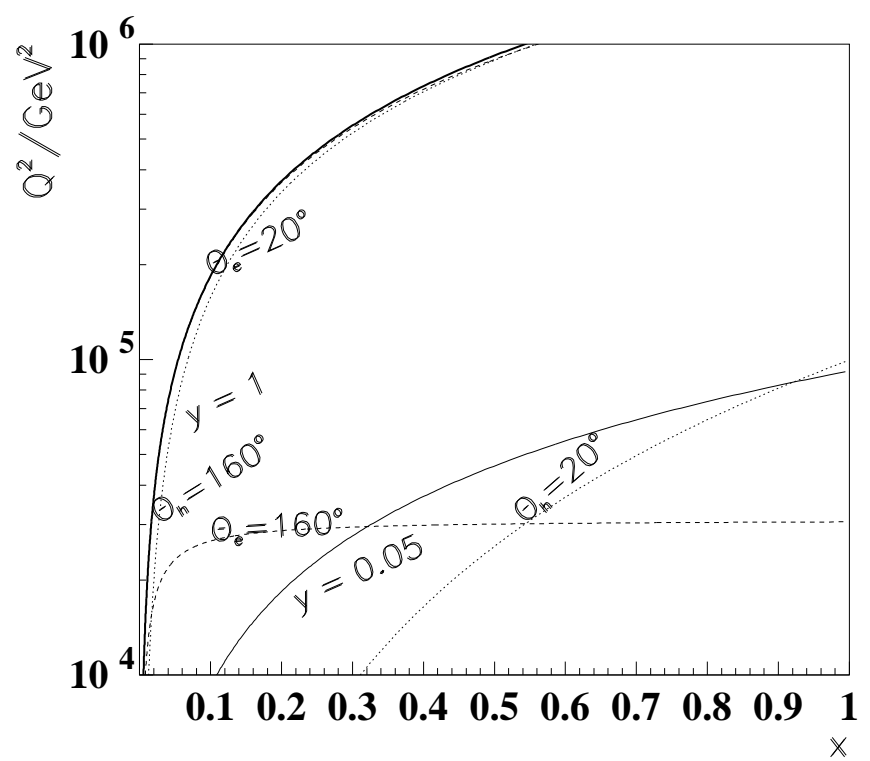

Figure 15: Kinematic region of a possible future ep collider using $500 \mathrm{GeV}$ electrons from TESLA and $920 \mathrm{GeV}$ protons from HERA. The line $y=0.05$ represents the upper kinematic limit of ep collisions in HERA. Since the TERA machine is symmetric in energy, it provides full containment of highly energetic electrons and hadrons at large $x$ and high $Q^{2}$ as indicated with the iso- $\theta$ lines at $20^{\circ}$ and $160^{\circ}$.

low $x$ acceptance by a factor of 20 and DIS data to $Q^{2} \simeq 500,000 \mathrm{GeV}^{2}$ and beyond, (Fig. 15). Saturation and sub-structure will be searched for in this extended range.

30 years after the pioneering SLAC ep experiments deep inelastic scattering still has an exciting future.

\section{Concluding Remarks}

HERA has opened the field of low $x$ physics which is governed by gluon interactions and which is far from being fully understood. The gluon momentum density at low $x$ is very large. This causes the structure function $F_{2}$ to rise at low $x$, it determines the longitudinal structure function to be large and the production cross section of heavy flavours to be sizeable. Increasing experimental precision leads to sensitive tests of QCD at higher orders perturbation theory. Most accurate simultaneous determinations are in reach of the gluon distribution and the strong interaction coupling constant with DIS data. Electroweak neutral and charged current structure functions provide new insights in the proton structure at high $x$. Measurements at $Q^{2} \simeq M_{Z}^{2}$ probe the proton nearly 100 times below the parton level reached three decades ago. 
It is a spectacular result that no substructure of leptons or quarks has been observed so far. At the same time significant progress is made with various fixed target and $p p$ experiments leading to deeper insight in the partonic structure of the proton. The gluon distribution at large $x$ is small but remains to be determined. The next step is in reach for tests of the inner proton structure down to $2 \cdot 10^{-19} \mathrm{~m}$. The outcome is unknown and deep inelastic physics therefore worth continuing effort.

It is a pleasure to thank John Jaros and co-organizers for an excellent Symposium. Many thanks are due to colleagues from the various DIS experiments for providing information and guidance in understanding their results. I have to thank too many individuals to be named here, members of the H1 Collaboration and its structure function group, physicists and engineers of the Zeuthen Silicon detector group, colleagues in the DIS99 conference committees, many theoretical and experimental physicists for useful discussions and reading the manuscript and also several known

physicists around the BCDMS Collaboration who introduced me to deep inelastic scattering years ago. Modern particle physics is a huge common effort of a large, mostly friendly community. Particular recognition is due to the youngest: I sincerely thank Vladimir Arkadov, Doris Eckstein, Alexander Glazov and in particular Rainer Wallny for efficient help in preparing this talk and exciting moments of joint research.

\section{References}

[1] E.D. Bloom et al., Phys. Rev. Lett. 23, 930 (1969);

M. Breidenbach et al., Phys. Rev. Lett. 23, 935 (1969).

[2] M. Yearin and R. Hofstadter, Phys. Rev. 110, 552 (1958).

[3] R.P. Feynman, Phys. Rev. Lett. 23, 935 (1969).

[4] J. Bjorken, Phys. Rev. 179, 15471969.

[5] J. Bjorken and E.A. Paschos, Phys. Rev. 185, 19751969.

[6] D. J. Fox et al., Phys. Rev. Lett. 33, 1504 (1974).

[7] D. J. Gross and F. Wilczek, Phys. Rev. D 9, 980 (1974);

H. Georgi and H. D. Politzer, Phys. Rev. D 9, 416 (1974).

[8] For a review see: P. Gallison, Rev. Mod. Phys. 55, 477 (1983).

[9] C. Y. Prescott et al., Phys. Lett. B 77, 347 (1978). 
[10] E. Derman, Phys. Rev. D 7, 2755 (1973).

[11] M. Klein and T. Riemann, Z. f. Phys. C 24, 151 (1984).

[12] A. Argento et al., BCDMS Collaboration, Phys. Lett. 140 B, 142 (1984).

[13] I. Abt et al., H1 Collaboration, Nucl.Phys. B 407, 515 (1993).

[14] M. Derrick et al., ZEUS Collaboration, Phys. Lett. B 316, 412 (1993).

[15] A. De Rujula, et al., Phys. Rev. D 10, 1649 (1974).

[16] G. Parisi and R. Petronzio, Phys. Lett. 62 B, 331 (1976),

V. Novikov et al., JETP Lett. 76, 341 (1976),

M. Glück and E. Reya, Nucl. Phys. B 130, 76 (1977).

[17] M. Glück, E. Reya and A. Vogt, Z. f. Phys. C 53, 127 (1992).

[18] J. Blümlein, Proc. Ringberg Workshop "Trends in HERA Physics 1999", eds. G. Grindhammer, B. Kniehl and G. Kramer, Springer, Berlin 1999, hepph/990949 (1999) and refs. cited therein.

[19] P. Marage, Inv. Talk, Proc. of the EPS Conf. Tampere, Finnland, 1999, to appear.

[20] G. Altarelli, R. Ball and S. Forte, CERN-Th/2000-010, hep-ph/00001157.

[21] Proc. of the 7th International Workshop on Deep Inelastic Scattering and QCD, Nucl. Phys. B (Proc. Suppl.) 79 (1999), ed. by J. Blümlein and T. Riemann.

[22] V. Shekelyan, Proc. Int. Symposium on Lepton-Photon Interactions, Hamburg 1997, World Scientific, Singapore 1998, ed. by A. De Roeck and A. Wagner.

[23] A. Levy, The Structure of the Troika, Taup 2531, hep-ph/9811462 (1998).

[24] A. Donnachie and P.V. Landshoff, Phys. Lett. B 437,408 (1998);

P.V. Landshoff in [21], p.204.

[25] C. Amelung, in [21], p.176 (1999).

[26] J.J. Sakurai and D. Schildknecht, Phys. Lett. B 40, 121 (1972).

[27] H. Abramowicz and A. Levy, DESY preprint 97-251 (1997).

[28] E. Bloom and F. Gilman, Phys. Rev. D 4, 2901 (1971).

[29] C. Keppel, in [21], p.182 (1999).

[30] A. De Rujula, H. Georgi and H.D. Politzer, Ann. Phys. 103, 3115 (1977). 
[31] W. Seligman et al., CCFR Collaboration, Phys. Rev. Lett. 79, 1213 (1997).

[32] M. Vakili et al., CCFR Collaboration, hep-ex/9905052 (1999).

[33] A.C. Benvenuti et al., BCDMS Collaboration, Z. f. Phys. C 63, 29 (1994).

[34] IHEP-JINR Collaboration, hep-ex/9905038 (1999);

A.V. Sidorov et al., in [21], p.99 (1999).

[35] A.T. Doyle, Proc. XXIX Int. HEP Conf., Vancouver, Canada, p. 193, World Scientific, Singapore, 1999, ed. by A. Astbury, D. Axen and J. Robinson.

[36] U.K. Yang, CCFR Collaboration, private communication.

[37] F. Boros et al., Phys. Lett. B 468, 161 (1999).

[38] V. Barone, C. Pascaud and F. Zomer, hep-ph/9907512 (1999).

[39] R. Gandhi et al., Phys. Rev. D 58, 093009 (1998).

[40] M. Glück, S. Kretzer and E. Reya, Astro. Part. Phys. 11, 327 (1998).

[41] J. Kwiecinski, A.D. Martin and A.M. Stasto, hep-ph/9905307 (1999).

[42] U.K. Yang et al., CCFR Collaboration, in [21], p.89 (1999).

[43] K. Ackerstaff et al., HERMES Collaboration, hep-ex/9910071 (1999).

[44] M. Klein, H1 Collaboration, Proc. XXIX Int. HEP Conf., Vancouver, Canada, p.819, World Scientific, Singapore, 1999, ed. by A. Astbury, D. Axen and J. Robinson.

[45] E. Zijlstra and W. van Neerven, Nucl. Phys. 383, 525 (1992).

[46] C. Adloff et al., H1 Collaboration, DESY 99-107, hep-ex/9908059 (1999), Eur. Phys. J. to appear.

[47] H1 Collaboration, Paper no.157b, submitted to the EPS Conf. Tampere, Finnland, 1999, presented by E. Rizvi.

[48] J. Breitweg et al., ZEUS Collaboration, DESY 99-056 (1999).

[49] J. Breitweg et al., ZEUS Collaboration, DESY 99-059 (1999).

[50] J. Breitweg et al., ZEUS Collaboration, Papers no.1549 and 558, submitted to the EPS Conf. Tampere, Finnland, 1999, presented by P. Koijman.

[51] A. Bodek and U.K. Yang, Phys. Rev. Lett. 82, 2467 (1999). 
[52] C. Boros, J.T. Lonergan and A.W. Thomas, Phys. Rev. Lett. 81, 4075 (1998).

[53] A. Bodek, in [21] p. 137 (1999).

[54] R. Towell, NuSea Collaboration, Inv. Talk, Proc. of the EPS Conf. Tampere, Finnland, 1999, to appear.

[55] C.A. Miller, in [21], p. 146 (1999).

[56] A. Szczurek and V. Uleshchenko, in [21], p. 149 (1999) and refs. cited therein.

[57] T. Adams et al., NuTeV Collaboration, hep-ex/9906038 (1999).

[58] W. Melnitchouk, hep-ph/9906488 (1999) and refs. cited therein.

[59] E. Witten, Nucl. Phys. 104, 445 (1976);

M.A. Shifman, A.I. Vainshtein and V.I. Zakharov, Nucl. Phys. 136, 157 (1978);

M. Glück and E. Reya, Phys. Lett. B 83, 999 (1979).

[60] J. Smith, in [21], p.124 (1999) and refs. cited therein;

for a review see: W. van Neerven, Lecture at the Zakopane Winter School, 1998.

[61] W.K. Tung, Proc. of the 5th International Workshop on Deep Inelastic Scattering and QCD, Chicago, USA (1997), p. 1410, ed. by J. Repond and D. Krakauer.

[62] J. Breitweg et al., ZEUS Collaboration, DESY 99-101, hep-ex/9908012 (1999).

[63] V.S. Fadin and L.N. Lipatov, Phys. Lett. B 429, 127 (1998);

M. Ciafaloni, in [21], p. 201 (1999) and refs. cited therein.

[64] R.S. Thorne, in [21], p. 210 (1999).

[65] A. Caldwell, Inv. Talk, DESY Theory Workshop, Hamburg, October 1997;

J. Breitweg et al., ZEUS Collaboration, Eur. Phys. J. C 7, 609 (1999).

[66] E. Gotsman, E. Levin and U. Maor, Nucl. Phys. 539, 535 (1999)

[67] C. Merino, A. B. Kaidalov and D. Petermann, hep-ph/9911331 (1999).

[68] E. Laenen et al., Nucl. Phys. B 392, 162 (1993);

S. Riemersma, J. Smith and W. van Neerven, Phys. Lett. B 347, 143 (1995).

[69] C. Pascaud and F. Zomer, LAL Orsay preprint, LAL/95-05 (1995).

[70] C. Adloff et al., H1 Collaboration, Nucl. Phys. B 545, 21 (1999).

[71] M.A. Shifman, A.I. Vainshtein and V.I. Zakharov, Phys. Lett. 65 B, 255 (1976). 
[72] M. Begel, in [21], p. 244 and ref. therein.

[73] F. Halzen and D.M. Scott, Phys. Rev. D 22, 1617 (1980).

[74] L. Apanasevich et al., Phys. Rev. Lett. 81, 2642 (1998).

[75] M. Botje, M.Klein and C. Pascaud, Proc. Int. Workshop on Future Physics at HERA, Hamburg 1996, Vol.1, p. 33, ed. by G. Ingelman, A. De Roeck and R. Klanner.

[76] M. Glück, E. Reya and A. Vogt, hep-ph/9806404 (1998).

[77] A. Martin et al., hep-ph/9907231 (1999).

[78] H.L. Lai et al., CTEQ Collaboration, hep-ph/9903282 (1999).

[79] M. Botje, in [21, p.111 (1999).

[80] S. Alekhin, Phys. Rev. D 59, 114016 (1999).

[81] A. Vogt, in [21], p.102 (1999).

[82] A. Milsztaijn and M. Virchaux, Phys. Lett. B 274, 221 (1992).

[83] W. van Neerven, in [21], p.36 (1999).

[84] A.L. Kataev, G. Parente and A.V. Sidorov, hep-ph/9905310 (1999).

[85] J. Santiago and F.J. Yndurain, hep-ph/9907387 (1999).

[86] P.W. Johnson and W. Tung, Phys. Rev. D16, 2769 (1977).

[87] L. Magnea and S. Forte, hep-ph/9910421 (1999).

[88] R. D. Ball and S. Forte, Phys. Lett. B 358, 365 (1995).

[89] A. De Roeck, M. Klein and Th. Naumann, Phys. Lett. B 385, 411 (1996).

[90] E. Levin, DESY preprint 99-113, hep-ph/9908379 (1999).

[91] A. Wagner, in [21], p.176 (1999).

[92] J. Morfin, in [21, p.664 (1999).

[93] S. Geer, Phys. Rev. D 57, 1 (1998).

[94] B. King, Proc. Muon Collider Workshop, Fermilab, Nov. 1997, hep-ex/9907033 (1999).

[95] M. Tigner, B.H. Wiik and F. Willeke, Proc. Part. Acc. Conf., San Francisco, Vol. 5, p. 2910 (1991). 